\title{
Anticipating integrals for a class of martingales
}

\author{
JIN MA, ${ }^{1}$ PHILIP PROTTER ${ }^{2 *}$ and JAIME SAN MARTIN ${ }^{3}$ \\ ${ }^{1}$ Department of Mathematics, Purdue University, West Lafayette IN 47907-1395, USA \\ ${ }^{2}$ Department of Mathematics and Statistics, Purdue University, West Lafayette IN 47907-1395, \\ USA \\ ${ }^{3}$ Facultad de Ciencias Físicas y Matemáticas, Universidad de Chile, Beauchef 850, Santiago, \\ Chile
}

An anticipating stochastic integral is proposed for 'normal martingales'. It agrees with the Skorohod integral in the Brownian case. A variational derivative of Malliavin type is also defined. An integration by parts formula is given which has some subtle and important differences from the formula in the Brownian case. The existence and uniqueness of solutions of linear stochastic differential equations with anticipating exogenous driving terms are also established.

Keywords: anticipating stochastic differential equation; anticipating stochastic integral; Azéma's martingale; homogeneous chaos; multiple stochastic integral; normal martingales; stochastic integration by parts; structure equation; variational derivative

1350-7265 (C) 1998 Chapman \& Hall

\section{Introduction}

In this paper we propose an anticipating integral for general martingales possessing two key properties: that $\langle M, M\rangle_{t}=t$; and that $M$ possesses the chaos representation property (CRP). Martingales having the first property have been called 'normal' by Dellacherie et al. (1992, p. 199) and are discussed in Section 2. Our integral is based on chaos expansion and as such it is analogous to the Skorohod integral as developed by Nualart and Pardoux (1988). When the integrand is predictable it reduces to the usual martingale integral as presented, for example, in Protter (1990); when $M$ is a Brownian motion it is exactly the Skorohod integral.

There are many similarities between our martingale anticipating integral and the Skorohod integral, but there are also some important (and non-trivial) differences. Many of these differences stem from one key fact: in the Brownian case $[B, B]_{t}=\langle B, B\rangle_{t}=t$, while in our case only $\langle M, M\rangle_{t}=t$, and $[M, M]_{t}$ is random; see Protter (1990) for all unexplained notation. For example, there are two ways to describe a variational derivative (also known as the 'Malliavin derivative' in the Brownian case), and they are equivalent in

\footnotetext{
*To whom correspondence should be addressed. e-mail: protter@math.purdue.edu
} 
the Brownian case but not in ours. This leads to some subtle differences, and null sets exist for measures arising from kernels on the product space $\Omega \times \mathbb{R}_{+}$.

Our paper is organized as follows. In Sections 3 and 4 we recall what chaos is, and in Section 4 we give the basic definitions of a variational derivative and an anticipating integral, and we establish some elementary properties which are analogues of the Brownian (Skorohod) case. Here there already arises a difference: in the Brownian case one can use the equivalence of the two definitions of the variational derivative to give elegant proofs (using the 'integration by parts' formula) right from the beginning - see the elegant presentation of Nualart (1995), for example - whereas such tools are not available for our integral.

In Section 5 we continue our study of properties of the Skorohod integral and we include a formula inspired by preliminary work of Russo and Vallois (1994, Theorem 5.3). Here the subtlety of the differences with the Brownian case begins to become readily apparent, and correct definitions with respect to reference measures need to be made.

In Section 6 we give some preliminary results on stochastic differential equations (SDEs). Our results are a far cry from the beautiful results already established for the Skorohod integral - see, for example, Buckdahn (1994) - but on the other hand they are very general in that they hold for any normal martingale $M$. Our method relies exclusively on the CRP of a normal martingale, therefore neither path regularity nor $L^{p}$ estimations of the anticipating integrals are required.

The reader will note that we do not include results establishing path regularity of the integral, and hence we are as yet unable to establish a change of variables formula. We hope to address these issues in future work.

\section{Normal martingales}

The following definition is from Dellacherie et al. (1992, pp. 199ff).

Definition 2.1. A martingale $M$ is called normal if $\langle M, M\rangle_{t}=t$.

In this paper we will be interested in normal martingales that have an extra property: the chaos representation property (see Section 3). Note that $[M, M]_{t}-\langle M, M\rangle_{t}=[M, M]_{t}-t$ is a martingale, and because the CRP implies the predictable representation property, we have that

$$
[M, M]_{t}=t+\int_{0}^{t} \varphi_{s} \mathrm{~d} M_{s}
$$

for some predictable $\varphi$. In other words, equation (2.1) is necessary if a martingale $M$ is normal and has the CRP. Emery (1989) first presented and studied equation (2.1) and has called it the structure equation. If one begins with the structure equation (2.1), then one can show that it has unique solutions under quite general conditions on $\varphi$ (see Meyer 1989; or Kurtz and Protter 1991, pp. 1044-1045). The simplest structure equations are those of Brownian motion and the compensated Poisson process, which are respectively: 


$$
[M, M]_{t}=t, \quad \text { and } \quad[M, M]_{t}=t+c M_{t}, \quad c>0 .
$$

An important special class was studied by Emery, which we write in differential form:

$$
\mathrm{d}[M, M]_{t}=\mathrm{d} t+\left(\alpha+\beta M_{t-}\right) \mathrm{d} M_{t} ; \quad M_{0}=x .
$$

Particularly if $\alpha=0$ and $-2 \leqslant \beta \leqslant 0$, Emery proved the important result that a solution $M$ of (2.2) is normal and has the CRP. This gives a whole family of normal martingales with the CRP for which our results apply. Note that $\beta=0$ is Brownian motion and $\beta=-1$ is Azéma's martingale; see, for example, Protter (1990, pp. 180-185) for a treatment of Azéma's martingale. When $\beta=-2, M$ is known as the 'parabolic martingale' with $\left|M_{t}\right|=\sqrt{ } t$ a.s., first studied by Protter and Sharpe (1979) and Barlow (1981). Vallois (1995) has recently demonstrated an interesting connection between the Brownian range process and parabolic martingales, thus showing that if $M_{t}=\sqrt{ } 2 B_{\theta(\sqrt{ } t)}$, where $B$ is standard Brownian motion and $\theta$ is the inverse of its range process, then $M$ is also a normal martingale with the CRP. It is worth pointing out that any solution $M$ of (2.2) has no continuous martingale part: that is, $[M, M]_{t}^{\mathrm{c}}=0$, for all $t$ (see Emery 1989). Finally, Russo and Vallois (1994) have extended Emery's results a little by considering the equation:

$$
\mathrm{d}[M, M]_{t}=\mathrm{d} t+\beta(t) M_{t-} \mathrm{d} M_{t} .
$$

They show that if $-2 \leqslant \beta(t) \leqslant 0$, for all $t$, then $M$ is again normal and has the CRP.

The preceding discussion hopefully indicates that there is a significant family of normal martingales having the CRP already known to exist, with the prospect of more.

\section{Multiple integrals and preliminaries}

Since the recent book of Dellacherie et al. (1992) gives a lovely treatment of multiple integrals for normal martingales, we do not give one here, but content ourselves with a definition. We let $\Sigma_{n}$ be an 'increasing simplex' of $\mathbb{R}_{+}^{n}$ :

$$
\Sigma_{n}=\left\{\left(t_{1}, \ldots, t_{n}\right) \in \mathbb{R}_{+}^{n}: 0<t_{1}<\ldots<t_{n}\right\},
$$

and we extend a function $f$ defined on $\Sigma_{n}$ by making $f$ symmetric on $\mathbb{R}_{+}^{n}$. We can then define

$$
I_{n}(f)=n ! \int_{\Sigma_{n}} f\left(t_{1}, \ldots, t_{n}\right) \mathrm{d} M_{t_{1}} \ldots \mathrm{d} M_{t_{n}} .
$$

This has the advantage of working with traditional adapted integrands for our martingale integrals. Note that the domain $\Sigma_{n}$ and its symmetrizations do not cover $\mathbb{R}_{+}^{n}$ : we are ignoring the diagonals. As Meyer (1976) pointed out, this ignores terms such as

$$
\int_{\left\{t_{1}=t_{2}\right\}} f\left(t_{1}, t_{2}\right) \mathrm{d} M_{t_{1}} \mathrm{~d} M_{t_{2}}=\int_{0}^{\infty} f(t, t) \mathrm{d}[M, M]_{t},
$$

which need not be trivial in our case. We avoid this problem by adopting the following convention: 
Convention 3.1. Functions $f$ defined on the simplex $\Sigma_{n}$ are automatically extended to $\mathbb{R}_{+}^{n}$ such that $f$ is symmetric and zero on the diagonals.

More precisely, let us define a class of functions for $n \in \mathbb{N}$ and $T=[0,1]$ by

$$
L_{s}^{2}\left(T^{n}\right)=\left\{f \in L^{2}\left(T^{n}\right) \mid f \text { is symmetric in all variables }\right\} .
$$

Note that elements in $L_{s}^{2}\left(T^{n}\right)$ are in fact equivalence classes, and we shall always choose a representative that vanishes on the 'diagonal' set: $\Delta_{n}=\left\{\left(t_{1}, \ldots, t_{n}\right) \mid \exists t_{i}=t_{j}, i \neq j\right\}$. Such a choice of representative will not affect our discussion when we are treating the iterated integral and its norm in $L^{2}\left(T^{n}\right)$, since in the former case the set $\Delta_{n}$ is never involved, and in the latter $\Delta_{n}$ is a null set under the Lebesgue measure. However, it will become crucial when some measure involving $\mathrm{d}[M, M]_{t}$ is considered, as in general $\mathrm{d}[M, M]_{t}$ will charge the Lebesgue null set.

The multiple integral with respect to $M$ defined in (3.2) will be considered defined for every $f \in L_{s}^{2}\left(T^{n}\right)$ (or $L^{2}\left(\Sigma_{n}\right)$ ); and it is known (see, for example, Meyer 1993) that for each $f \in L_{s}^{2}\left(T^{n}\right)$

$$
\left\|I_{n}(f)\right\|_{L^{2}(\Omega)}^{2}=(n !)^{2}\|f\|_{L^{2}\left(\Sigma_{n}\right)}^{2}=n !\|f\|_{L^{2}\left(T^{n}\right)}^{2} .
$$

Definition 3.2. Let $\mathscr{G}=\sigma\left\{M_{t} ; t \geqslant 0\right\}$, the $\sigma$-field generated by a (normal) martingale $M$. Let $\mathscr{H}_{n}$ be the nth homogeneous chaos, $\mathscr{H}_{n}=I_{n}(f)$, where $f$ ranges over all $f \in L^{2}\left(\Sigma_{n}\right)$. If $L^{2}(\mathscr{G}, \mathrm{d} P)=\oplus_{n=0}^{\infty} \mathscr{H}_{n}$ (the direct sum), then we say $M$ possesses the chaos representation property.

Remark 3.3. The hypothesis $\langle M, M\rangle_{t}=t$ has been used to define multiple integrals in a nice way, which in turn are needed to define the CRP. One could require only $\mathrm{d}\langle M, M\rangle_{t} \ll \mathrm{d} t$ to define the multiple integral, but if $\langle M, M\rangle_{t}$ were random one would lose the property that different chaoses are orthogonal. Meyer (1976, p. 325) also points out that one could assume that $\langle M, M\rangle_{t}=c(t)$, where $c(\cdot)$ is non-random, but we do not pursue such generality here, partially because to date there are no known interesting examples of martingales $M$ such that $\langle M, M\rangle_{t}=c(t) \neq t$ and which have the CRP.

In what follows we shall always assume that a normal martingale $M$ with the CRP is given on the probability space $(\Omega, \mathscr{F}, P)$, and that $\mathscr{F}$ is generated by $M$. Thus, for any random variable $F \in L^{2}(\mathscr{F}, \mathrm{d} P) \quad\left(=L^{2}(\Omega)\right)$ we have by the CRP that there exists a sequence of functions $f_{n} \in L_{s}^{2}\left(T^{n}\right), n=1,2, \ldots$, such that $F=\sum_{n=0}^{\infty} I_{n}\left(f_{n}\right)$. Hence

$$
\infty>\|F\|_{L^{2}(\Omega)}^{2}=\sum_{n=0}^{\infty}\left\|I_{n}\left(f_{n}\right)\right\|_{L^{2}(\Omega)}^{2}=\sum_{n=0}^{\infty} n !\left\|f_{n}\right\|_{L^{2}\left(T^{n}\right)}^{2} .
$$

We shall also use the following notation throughout this paper. If $f \in L^{2}\left(T^{n}\right)$ and $g \in L^{2}\left(T^{m}\right)$, we denote $f \otimes g \in L^{2}\left(T^{n+m}\right)$ by

$$
f \otimes g\left(t_{1}, \ldots, t_{n} ; s_{1}, \ldots, s_{m}\right)=f\left(t_{1}, \ldots, t_{n}\right) g\left(s_{1}, \ldots, s_{m}\right) ;
$$


and if $I \subseteq T=[0,1]$, and $k \in \mathbb{N}$, we denote by $1_{I}^{\otimes k}$ a function in $L^{2}\left(T^{k}\right)$ such that

$$
1_{I}^{\otimes k}\left(t_{1}, \ldots, t_{k}\right)=\prod_{i=1}^{k} 1_{I}\left(t_{i}\right) .
$$

\section{A derivative operator and an anticipating integral}

Consider the following subset $\mathscr{D} \subset L^{2}(\Omega)$ :

$$
\mathscr{D}:=\left\{F=\sum_{n=0}^{\infty} I_{n}\left(f_{n}\right) \mid \sum_{n=0}^{\infty} n n !\left\|f_{n}\right\|_{n}^{2}<\infty\right\},
$$

where $\|\cdot\|_{n}:=\|\cdot\|_{L^{2}\left(T^{n}\right)}$. To be consistent with the usual notation in the literature (see, for example, Nualart and Pardoux 1988), we shall write $\mathscr{D}=\mathbb{D}_{1,2}$. It is easily seen that $\mathbb{D}_{1,2}$ is dense in $L^{2}(\Omega)$, since every element in the finite Hilbert sum of chaoses belongs to $\mathbb{D}_{1,2}$. The derivative operator is analogous to what is often called the Malliavin derivative in the Brownian case, and it is defined as a linear operator $D: \mathbb{D}_{1,2} \subset L^{2}(\Omega) \rightarrow L^{2}(T \times \Omega)$, by

$$
D_{t} F:=\sum_{n=1}^{\infty} n I_{n-1}\left(f_{n}(\cdot, t)\right), \quad t \in[0,1],
$$

whenever $F$ has the chaos expansion $F=\sum_{n=0}^{\infty} I_{n}\left(f_{n}\right)$. It is easy to see that

$$
\begin{aligned}
\left\|D_{t} F\right\|_{L^{2}(T \times \Omega)}^{2} & =\int_{0}^{1}\left\|\sum_{n=1}^{\infty} n I_{n-1}\left(f_{n}(\cdot, t)\right)\right\|_{L^{2}(\Omega)}^{2} \mathrm{~d} t \\
& =\int_{0}^{1} \sum_{n=1}^{\infty} n^{2}(n-1) !\|f(\cdot, t)\|_{L^{2}\left(T^{n-1}\right)}^{2} \mathrm{~d} t \\
& =\sum_{n=1}^{\infty} n n ! \int_{0}^{1}\|f(\cdot, t)\|_{L^{2}\left(T^{n-1}\right)}^{2} \mathrm{~d} t=\sum_{n=1}^{\infty} n n !\|f\|_{n}^{2}<\infty,
\end{aligned}
$$

for all $F \in \mathbb{D}_{1,2}$. Note that since our definition is analogous to the one normally used for the Brownian chaos expansion, we can derive several properties using exactly the same methods as those used in the Brownian case. Surprisingly, however, the definition is not compatible with a Sobolev space structure, as is of course the case for Brownian motion. We shall explain this via an example at the end of this section. Here we give two properties of the operator $D$ for ready reference.

Lemma 4.1. (1) Suppose that $F \in L^{2}(\Omega)$. Then $D_{t} F=0$, for all $t \in[0,1]$ if and only if $F$ is a constant (non-random).

(2) Suppose that $F \in L^{2}(\Omega)$ and is $\mathscr{F}_{t}$-measurable. Then $D_{s} F=0$ for all $s>t$. 
Proof. This is identical to the Brownian case, so we omit it.

We now turn to the definition of an anticipating integral, which is analogous to that of the Skorohod integral in the Brownian case. Note that since $D$ is a densely defined operator, we can define its adjoint operator, denoted by $\delta$, in the usual way. That is to say, let

$$
\mathscr{D}^{*}:=\mathscr{D}(\delta):=\left\{G \in L^{2}(T \times \Omega): \exists C>0,\left|\mathrm{E} \int_{0}^{1} G(t, \cdot) D_{t} F \mathrm{~d} t\right| \leqslant C\|F\|, \forall F \in \mathbb{D}_{1,2}\right\},
$$

and the adjoint operator $\delta: L^{2}(T \times \Omega) \mapsto L^{2}(\Omega)$ is defined by the equation:

$$
\mathrm{E}(\delta(G) F)=\mathrm{E} \int_{0}^{1} G(t, \cdot) D_{t} F \mathrm{~d} t, \quad \forall F \in \mathbb{D}_{1,2}, G \in \mathscr{D}^{*} .
$$

Since every element in $L^{2}(T \times \Omega)$ also has a chaos expansion, we can write $G(t, \cdot)=$ $\sum_{n=0}^{\infty} I_{n}\left(g_{n}(\cdot, t)\right)$, where the $g_{n}$ are deterministic functions, jointly measurable in all variables, and symmetric in the first $n$ variables. Following the same arguments as those in Nualart (1995), one can show that the set $\mathscr{D}^{*}$ is dense in $L^{2}(T \times \Omega)$ and that

$$
\mathscr{D}^{*}=\left\{G(t, \cdot)=\sum_{n=0}^{\infty} I_{n}\left(g_{n}(\cdot, t)\right): \sum_{n=0}^{\infty}(n+1) !\left\|\bar{g}_{n}^{\mathrm{s}}\right\|_{L^{2}\left(T^{n+1}\right)}<\infty\right\},
$$

where $\bar{g}^{\mathrm{s}}$ denotes the symmetrization of $g$ in all variables. In other words, the set $\mathscr{D}^{*}$ is of the same form as the usual notion $\operatorname{Dom}(\delta)$ in the literature on the Skorohod integral (cf., for example, Nualart 1995, Proposition III.3), and we will therefore not distinguish the two from here on. Consequently, as the adjoint operator of $D, \delta$ is a densely defined, closed operator, which in turn shows that the operator $D$ is closable (in fact, it is not too hard to show that $D$ is closed by definition). We have the following definition.

Definition 4.2. The adjoint operator of $D$, denoted by $\delta: L^{2}(T \times \Omega) \mapsto L^{2}(\Omega)$, is called the anticipating integral of the element in $\mathscr{D}^{*}=\operatorname{Dom}(\delta)$. Furthermore, for any $u \in \operatorname{Dom}(\delta)$, we denote

$$
\delta(u)=\int_{0}^{1} u_{s} \delta M_{s}
$$

Also, we can define the set $\mathbb{1}^{1,2}$ as usual by

$$
\mathbb{L}^{1,2}:=\left\{u_{t}=\sum_{n=0}^{\infty} I_{n}\left(f_{n}(\cdot, t)\right) \mid \sum_{n=0}^{\infty} n n !\left\|f_{n}\right\|_{L^{2}\left(T^{n+1}\right)}^{2}<\infty\right\} .
$$

Noting that $\sum n n !\left\|f_{n}\right\|_{n+1}^{2}<\infty$ is equivalent to $\sum(n+1) !\left\|f_{n}\right\|_{n+1}^{2}<\infty$, and the obvious inequality

$$
\left\|\overline{f_{n}} \mathrm{~s}\right\|_{L^{2}\left(T^{n+1}\right)} \leqslant\left\|f_{n}\right\|_{L^{2}\left(T^{n+1}\right)},
$$


we have $\mathbb{1}^{1,2} \subset \operatorname{Dom}(\delta)$, as in the Brownian case. The following results for our anticipating integral can also be proved using exactly the same arguments as those used, for example, in Nualart (1995). We give only the statements.

Proposition 4.3. (1) Suppose that $u \in \operatorname{Dom}(\delta)$, and that $u$ has chaos expansion $u_{t}=$ $\sum_{n=0}^{\infty} I_{n}\left(g_{n}(\cdot, t)\right)$, where $g(\cdot, t) \in L_{s}^{2}\left(T^{n}\right)$, for almost all $t \in T$. Then

$$
\delta(u)=\sum_{n=0}^{\infty} I_{n+1}\left(\bar{g}_{n}^{\mathrm{s}}\right) .
$$

(2) Suppose that $u, v \in \mathbb{L}^{1,2}$. Then

$$
\mathrm{E}(\delta(u) \delta(v))=\mathrm{E} \int_{0}^{1} u_{s} v_{s} \mathrm{~d} s+\mathrm{E} \int_{0}^{1} \int_{0}^{1} D_{s} u_{t} D_{t} v_{s} \mathrm{~d} s \mathrm{~d} t .
$$

In particular, if $u=v \in \mathbb{L}^{1,2}$, then

$$
\mathrm{E}\left(\delta(u)^{2}\right)=\mathrm{E} \int_{0}^{1} u_{s}^{2} \mathrm{~d} s+\mathrm{E} \int_{0}^{1} \int_{0}^{1} D_{s} u_{t} D_{t} u_{s} \mathrm{~d} s \mathrm{~d} t .
$$

(3) Suppose that $u$ is a process in the space $\mathbb{L}^{1,2}$ such that for almost all $t \in[0,1]$, $D_{t} u$. $\in \operatorname{Dom}(\delta)$, and there is a version of the process $\left\{\int_{T}\left(D_{t} u_{s}\right) \delta M_{s} ; t \geqslant 0\right\}$ which is in $L^{2}(T \times \Omega)$. Then $\delta(u) \in \mathbb{D}_{1,2}$, and

$$
D_{t}(\delta(u))=\int_{0}^{1}\left(D_{t} u_{s}\right) \delta M_{s}+u_{t}
$$

A natural question now is whether the definition of our anticipating integral is a generalization of the usual Itô-type stochastic integral. Note that the proof in the Brownian case of this result (see, for example, Nualart 1995) uses the fact that any adapted $L^{2}$ process can be approximated by elementary adapted processes; and that the Skorohod and Itô integrals coincide on elementary adapted processes. The latter result relies on an integration by parts formula which in our case is more complicated and not easily applicable, as we shall see in the next section. Therefore the usual Brownian technique does not seem to apply. Our technique uses only the CRP (and not integration by parts), so it also gives an alternative (new) proof in the Brownian case.

Proposition 4.4. Suppose that $u \in L^{2}(T \times \Omega)$ is predictable. Then $u \in \operatorname{Dom}(\delta)$ and

$$
\delta(u)=\int_{0}^{1} u_{t} \mathrm{~d} M_{t}
$$

where the right-hand side above is in the semimartingale (or 'Itô') sense. 
Proof. Let us first assume that $u_{t}=I_{n}\left(f_{n}(\cdot, t)\right)$, where $f_{n}$ is some $L^{2}$-function, symmetric in the first $n$ variables. Since $u$ is predictable, we have

$$
u_{t}=n ! \int_{0}^{t}\left\{\int_{t_{1}<\ldots<t_{n}} f_{n}\left(t_{1}, \ldots, t_{n}, t\right) \mathrm{d} M_{t_{1}} \ldots \mathrm{d} M_{t_{n-1}}\right\} \mathrm{d} M_{t_{n}}=I_{n}\left(f_{n}(\cdot, t) 1_{[0, t)}^{\otimes n}\right) .
$$

Now by definition,

$$
\begin{aligned}
\delta(u) & =I_{n+1}\left(\overline{f_{n}(\cdot, *) 1_{[0, *)}^{\otimes n}(\cdot)^{\mathrm{s}}}\right) \\
& =(n+1) ! \int_{0}^{1}\left\{\int_{t_{1}<\ldots<t_{n}<t} \overline{f_{n}\left(t_{1}, \ldots, t_{n}, t\right) 1_{[0, t)}^{\otimes n}\left(t_{1}, \ldots, t_{n}\right)^{\mathrm{s}}} \mathrm{d} M_{t_{1}} \ldots \mathrm{d} M_{t_{n}}\right\} \mathrm{d} M_{t},
\end{aligned}
$$

where '.' stands for the first $n$ variables, and ' $*$ ' stands for the last variable; $\overline{f_{n}(\cdot, *) 1_{[0, *)}^{\otimes n}(\cdot)^{\mathrm{s}}}$ is the symmetrization of $f_{n}(\cdot, *) 1_{[0, *)}^{\otimes n}(\cdot)$ in all variables. Note that since $f_{n}$ is symmetric in the first $n$ variables, a little computation shows that on the set $\Sigma_{n+1}=\left\{t_{1}<t_{2}<\ldots<t_{n}<t\right\}$, we have

$$
\begin{aligned}
\overline{f_{n}\left(t_{1}, \ldots, t_{n}, t\right) 1_{[0, t)}^{\otimes n}\left(t_{1}, \ldots, t_{n}\right)^{\mathrm{s}}} & =\frac{1}{(n+1) !} \sum_{\sigma_{n}} f_{n}\left(\sigma_{n}(1), \ldots, \sigma_{n}(n), t\right) \\
& =\frac{1}{(n+1)} f_{n}\left(t_{1}, \ldots, t_{n}, t\right),
\end{aligned}
$$

where $\sigma_{n}$ runs over all the permutations of $\{1, \ldots, n\}$. Thus

$$
\delta(u)=n ! \int_{0}^{1}\left\{\int_{t_{1}<\ldots<t_{n}<t} f_{n}\left(t_{1}, \ldots, t_{n}, t\right) \mathrm{d} M_{t_{1}} \ldots \mathrm{d} M_{t_{n}}\right\} \mathrm{d} M_{t}=\int_{0}^{1} u_{t} \mathrm{~d} M_{t} .
$$

Now let us suppose that $u_{t}=\sum_{n=0}^{\infty} I_{n}\left(f_{n}(\cdot, t)\right) \in L^{2}(T \times \Omega)$, that is,

$$
\mathrm{E} \int_{0}^{1}\left|u_{t}\right|^{2} \mathrm{~d} t=\sum_{n=0}^{\infty} n ! \int_{0}^{1}\left\|f_{n}(\cdot, t)\right\|_{n}^{2} \mathrm{~d} t=\sum_{n=0}^{\infty} n !\left\|f_{n}\right\|_{n+1}^{2}<\infty .
$$

Since $u_{t}$ is predictable, it can be easily checked that every $I_{n}\left(f_{n}(\cdot, t)\right)$ must have the form $I_{n}\left(f_{n}(\cdot, t)\right)=I_{n}\left(f_{n}(\cdot, t) 1_{[0, t)}^{\otimes n}\right)$. Therefore, using the same argument as before, we have

$$
I_{n+1}\left(\overline{f_{n}} \mathrm{~s}\right)=\delta\left(I_{n}\left(f_{n}(\cdot, \cdot)\right)\right)=\int_{0}^{1} I_{n}\left(f_{n}(\cdot, t)\right) \mathrm{d} M_{t},
$$

and

$$
\begin{aligned}
& (n+1) !\left\|\overline{f_{n}} \mathrm{~s}\right\|_{n+1}^{2}=\mathrm{E}\left|I_{n+1}\left(\overline{f_{n}} \mathrm{~s}\right)\right|^{2}=\mathrm{E} \int_{0}^{t}\left|I_{n}\left(f_{n}(\cdot, t)\right)\right|^{2} \mathrm{~d} t=n ! \int_{0}^{t}\left\|f_{n}(\cdot, t)\right\|_{n}^{2} \mathrm{~d} t \\
& =n !\left\|f_{n}\right\|_{n+1}^{2} \text {. }
\end{aligned}
$$


Thus (4.7) gives that

$$
\sum_{n=0}^{\infty}(n+1) !\left\|\overline{f_{n}} \mathrm{~s}\right\|_{n+1}^{2}=\sum_{n=0}^{\infty} n !\left\|f_{n}\right\|_{n+1}^{2}<\infty,
$$

hence $u \in \operatorname{Dom}(\delta)$, and by Proposition 4.3(1),

$$
\delta(u)=\sum_{n=0}^{\infty} I_{n+1}\left(\overline{f_{n}} \mathrm{~s}\right)=\int_{0}^{1}\left\{\sum_{n=0}^{\infty} I_{n}\left(f_{n}(\cdot, t)\right)\right\} \mathrm{d} M_{t}=\int_{0}^{1} u_{t} \mathrm{~d} M_{t},
$$

proving the proposition.

\subsection{An Ocone-Haussmann-Clark type formula}

Let $M$ be a normal martingale with the CRP. For any $F \in L^{2}(\Omega)$, we can write

$$
F=\sum_{n=0}^{\infty} I_{n}\left(f_{n}\right)=\mathrm{E}(F)+\sum_{n=1}^{\infty} I_{n}\left(f_{n}\right)=\mathrm{E}(F)+\int_{0}^{t} u_{t} \mathrm{~d} M_{t},
$$

where $u$ is a predictable process given by

$$
u_{t}=\sum_{n=1}^{\infty} n ! \int_{t_{1}<t_{2}<\ldots<t_{n-1}<t} f_{n}\left(t_{1}, \ldots, t_{n-1}, t\right) \mathrm{d} M_{t_{1}} \ldots \mathrm{d} M_{t_{n-1}} .
$$

In the Brownian case, the predictable process $u$ can be further described using the 'Malliavin derivative' operator, known as the Ocone-Haussmann-Clark formula (see, for example, Ocone 1984). Our definition of the 'Malliavin derivative' operator $\left\{D_{t}\right\}_{t \geqslant 0}$ also allows us to derive an analogue of that formula, as we shall see in the following theorem.

Theorem 4.5. Let $M$ be a normal martingale with the $C R P$, and let $F \in L^{2}(\Omega)$. If $F \in \mathbb{D}_{1,2}$ then

$$
F=\mathrm{E}(F)+\int_{0}^{1} \mathrm{p}\left(D_{t} F\right) \mathrm{d} M_{t},
$$

where ${ }^{\mathrm{p}} H_{t}$ denotes the predictable projection of a process $H$.

Proof. Comparing (4.10) with (4.8), we see that we need only show $u_{t}={ }^{\mathrm{p}}\left(D_{t} F\right)$. Note that $u$ is already predictable, so if $u .={ }^{\circ}(D . F)$, the optional projection of $D . F$, then we would have ${ }^{\mathrm{p}}\left(D_{t} F\right)={ }^{\mathrm{p}}\left({ }^{\mathrm{o}}\left(D_{t} F\right)\right)={ }^{\mathrm{p}}\left(u_{t}\right)=u_{t}, \forall t \in[0,1]$, and we are done. Therefore it suffices to show that $u$ is the optional projection of D.F, or equivalently (see Dellacherie 1972),

$$
u_{\tau}=\mathrm{E}\left\{D_{\tau} F \mid \mathscr{F}_{\tau}\right\}, \quad \text { for any stopping time } \tau \in[0,1],
$$

where $D_{\tau} F$ is defined to be $\left.D_{t} F\right|_{t=\tau}$. To this end, we first note that by definition we have

$$
D_{t} F=\sum_{n=1}^{\infty} n I_{n-1}\left(f_{n}(\cdot, t)\right)=\sum_{n=1}^{\infty} n ! \int_{0}^{1} h_{n}\left(t_{n-1}, t\right) \mathrm{d} M_{t_{n-1}},
$$




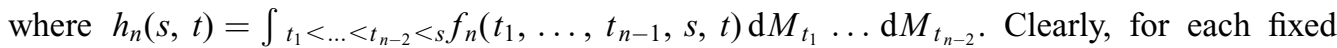
$t \in[0,1], h_{n}(\cdot, t)$ is predictable, so if we define $M_{n}(r, t):=\int_{0}^{r} h_{n}(s, t) \mathrm{d} M_{s}, r \in[0,1]$, then $M_{n}(\cdot, t)$ is a martingale with parameter $t \in[0,1]$. Thus for any fixed $t \in[0,1]$ and any stopping time $\tau \in[0,1]$, one has $\mathrm{E}\left\{M_{n}(1, t)-M_{n}(\tau, t) \mid \mathscr{F}_{\tau}\right\}=0$. By a standard monotone class argument, one can show that for any stopping time $\tau \in[0,1]$,

$$
\mathrm{E}\left\{M_{n}(1, \tau)-M_{n}(\tau, \tau) \mid \mathscr{F}_{\tau}\right\}=0 \quad \text { a.s. }
$$

Further, recalling that $f_{n}(\cdot, t, t)=0$, we have by (4.9) that $\sum_{n=1}^{\infty} n ! M_{n}(t, t)=u_{t}, \forall t$, a.s. Consequently, we see from (4.12) and (4.13) that, for any stopping time $\tau \in[0,1]$,

$$
\mathrm{E}\left\{D_{\tau} F \mid \mathscr{F}_{\tau}\right\}=\sum_{n=1}^{\infty} n ! \mathrm{E}\left\{M_{n}(1, \tau) \mid \mathscr{F}_{\tau}\right\}=\sum_{n=1}^{\infty} n ! \mathrm{E}\left\{M_{n}(\tau, \tau) \mid \mathscr{F}_{\tau}\right\}=u_{\tau} \quad \text { a.s., }
$$

where the last equality is due to the predictability of $u$. This proves (4.11), whence the theorem.

We remark that in the Brownian case this result is customarily stated more simply by writing $u_{t}=\mathrm{E}\left\{D_{t} F \mid \mathscr{F}_{t}\right\}$, for fixed $t$. It is implicit that in such an expression one really means that $u_{t}$ is the optional projection of $D_{t} F$.

\subsection{Indefinite integrals}

We now study the possibility of defining indefinite anticipating integrals. Note that even in the Brownian case, it is not true that for any $u \in \operatorname{Dom}(\delta)$, the process $\left\{u_{s} 1_{[0, t]}(s)\right.$ : $s \in[0,1]\} \in \operatorname{Dom}(\delta)$ (see Nualart and Pardoux 1988). We have the following definition.

Definition 4.6. Suppose that the process $u \in \operatorname{Dom}(\delta)$ is such that for any $t \in[0,1]$, we have that $u \cdot 1_{[0, t]}(\cdot) \in \operatorname{Dom}(\delta)$. Then the indefinite anticipating stochastic integral of $u$ on $[0, t]$ is defined by

$$
\int_{0}^{t} u_{s} \delta M_{s}:=\delta\left(u 1_{[0, t]}\right)=\int_{0}^{1} u_{s} 1_{[0, t]}(s) \mathrm{d} M_{s} .
$$

The following lemma gives a description of the class of processes in $\operatorname{Dom}(\delta)$ for which the indefinite integral exists.

Lemma 4.6. Suppose that $u \in \operatorname{Dom}(\delta)$, and $u_{s}=\sum_{n} I_{n}\left(f_{n}(\cdot, s)\right)$. Then for any $t \in[0,1]$, the process $u \cdot 1_{[0, t]}(\cdot) \in \operatorname{Dom}(\delta)$ if and only if

$$
\sum_{n=0}^{\infty}(n+1) !\left\|\overline{f_{n}(\cdot, *) 1_{[0, t]}(*)^{s}}\right\|_{n+1}^{2}<\infty .
$$

Consequently, a sufficient condition for the process $u \cdot 1_{[0, t]}(\cdot)$ to belong to $\operatorname{Dom}(\delta)$ is that $u \in \mathbb{L}^{1,2}$. 
Proof. The proof for (4.14) is direct, by using the definition. As for the second assertion, it suffices to recall the definition of $\mathbb{\complement}^{1,2}$ and the fact that

$$
\left\|\overline{f_{n}(\cdot, *) 1_{[0, t]}(*)^{\mathrm{s}}}\right\|_{n+1}^{2} \leqslant\left\|f_{n}(\cdot, *) 1_{[0, t]}(*)\right\|_{n+1}^{2} \leqslant\left\|f_{n}\right\|_{n+1}^{2} .
$$

To end this section we give an example which shows that one cannot define the derivative operator in the usual way in our case to obtain a Sobolev space structure for the space $\mathbb{D}_{1,2}$. In fact, the example somehow shows that the two definitions (Sobolev space and chaos expansion) are compatible if and only if the process $[M, M]$ is deterministic.

Example. Consider a symmetric function $f(s, t)=1_{(a, b]}(s) 1_{(a, b]}(t)$. The second chaos $I_{2}(f)$ can be computed as

$$
\begin{aligned}
I_{2}(f) & =2 ! \int_{0<s<t \leqslant 1} f(s, t) \mathrm{d} M_{s} \mathrm{~d} M_{t}=2 \int_{a}^{b} \int_{a}^{t-} \mathrm{d} M_{s} \mathrm{~d} M_{t}=2 \int_{a}^{b}\left(M_{t-}-M_{a}\right) \mathrm{d} M_{t} \\
& =\left(M_{b}-M_{a}\right)^{2}-\left\{[M, M]_{b}-[M, M]_{a}\right\} .
\end{aligned}
$$

Here, the last equality is due to Itô's formula. Now consider the function $F\left(x_{1}, x_{2}\right)=$ $\left(x_{2}-x_{1}\right)^{2}$, and define a smooth functional $\xi=F\left(M_{a}, M_{b}\right)$. Let us define the derivative $D_{t} \xi$ in a way analogous to one of the equivalent definitions in the Brownian case:

$$
\begin{aligned}
D_{t} \xi & =D_{t}\left(M_{b}-M_{a}\right)^{2}=\frac{\partial F}{\partial x_{1}}\left(M_{a}, M_{b}\right) 1_{[0, a]}(t)+\frac{\partial F}{\partial x_{2}}\left(M_{a}, M_{b}\right) 1_{[0, b]}(t) \\
& =2\left(M_{b}-M_{a}\right) 1_{(a, b]}(t) .
\end{aligned}
$$

However, by our definition

$$
D_{t} I_{2}(f)=2 I_{1}(f(\cdot, t))=2 \int_{0}^{1} 1_{(a, b]}(s) \mathrm{d} M_{s} \cdot 1_{(a, b]}(t)=2\left(M_{b}-M_{a}\right) 1_{(a, b]}(t) .
$$

We can substitute this into (4.16) and compare it with (4.15) to see that the two definitions coincide if and only if $D_{t}\left\{[M, M]_{b}-[M, M]_{a}\right\}=0$, for all $t \in T$. By Lemma 4.1(1), this means that $[M, M]_{b}-[M, M]_{a}$ must be constant. If we look at the structure equation (2.1), this amounts to saying that $\varphi \equiv 0$, therefore the two definitions are in contradiction and cannot hold simultaneously unless $M=B$, Brownian motion.

\section{Integration by parts formulae}

In this section we study an integration by parts formula given in Theorem 5.4. This formula differs from the one in the Brownian case in an important way, since it explicitly involves $[M, M]_{t}$, whose role is hidden in the Brownian case (in which $[M, M]_{t}=t$ ). Another difference is more subtle, in that one has to be careful what versions of the multiple integrals one takes. A key step in this direction was taken by Russo and Vallois (1994), which is 
presented here in Theorem 5.2; one difference in our treatment of Theorem 5.2 is that we take care to make precise which versions of the multiple integrals we are using. It is worth pointing out here that because in our case $\mathbb{D}_{1,2}$ does not have a Sobolev space structure, as we mentioned in the previous section, all the proofs of these properties will depend solely on the chaos expansion. Therefore they are sometimes more complicated than those of the Brownian case.

Let us first introduce some notation. Define, for each $n \in \mathbb{N}$, a vector space

$$
S_{n}:=\operatorname{span}\left\{\overline{\otimes^{n} 1_{I^{i}}(\cdot)^{\mathrm{s}}} \mid I^{i}=\left(a_{i}, b_{i}\right] ; a_{1}<b_{1} \leqslant a_{2}<b_{2} \leqslant \ldots \leqslant a_{n}<b_{n}\right\} .
$$

It is clear that $S_{n}$ is dense in both $L^{2}\left(\Sigma_{n}\right)$ and $L_{s}^{2}\left(T^{n}\right)$. Define, for $f, g \in L^{2}\left(T^{n}\right)$,

$$
\langle f, g\rangle_{n}=\int_{T^{n}} f\left(t_{1}, \ldots, t_{n}\right) g\left(t_{1}, \ldots, t_{n}\right) \mathrm{d} t_{1} \ldots \mathrm{d} t_{n} .
$$

We define a measure $\mu$ on the space $T \times \Omega$ in terms of the underlying martingale $M$ (known as the Doléans-Dade measure): for $t \in[0,1]$ and $B \in \mathscr{F}$,

$$
\mu([0, t) \times B):=\mathrm{E}\left\{1_{B}[M, M]_{t}\right\} .
$$

Then it is clear that $L^{2}(\mathrm{~d} \mu)=L^{2}(\mathrm{~d} t \times \mathrm{d} P)$ if and only if $[M, M]_{t}=t$. In other words, in the Brownian case $L^{2}(\mathrm{~d} \mu)$ is superfluous. Let us denote, for any $F, G \in L^{2}(\mathrm{~d} \mu)$,

$$
\langle F, G\rangle_{L^{2}(\mathrm{~d} \mu)}=\mathrm{E} \int_{0}^{1} F_{t} G_{t} \mathrm{~d}[M, M]_{t},
$$

and, for $n, m \in \mathbb{N}$ and $f \in S_{n}, g \in S_{m}$,

$$
(f, g)_{n, m}=\left\langle I_{n-1}(f(\cdot, \cdot)), I_{m-1}(g(\cdot, \cdot))\right\rangle_{L^{2}(\mathrm{~d} \mu)} .
$$

When $n=m$, we write $(f, g)_{n, n}=(f, g)_{n}$. We first give a lemma whose proof can be found in Russo and Vallois (1994).

Lemma 5.1. Suppose that $f \in S_{n}$ and $g \in S_{1}$. Then

$$
I_{n}(f) I_{1}(g)=I_{n+1}(f \otimes g)+n \int_{0}^{\infty} I_{n-1}\left(f(\cdot, t) g(t) \mathrm{d}[M, M]_{t} .\right.
$$

Next, we prove the following isometry property:

Lemma 5.2. If $f \in S_{n}$ and $g \in S_{m}, n, m \in \mathbb{N}$, then

$$
(f, g)_{n, m}= \begin{cases}0, & n \neq m ; \\ (n-1) !\langle f, g\rangle_{n}, & n=m .\end{cases}
$$

Proof. We split the proof into two separate cases.

Case $1(n=m)$. It suffices to consider the case when $f=g$, as the general case can be derived by polarization. In other words, we shall prove that

$$
(f, f)_{n}=(n-1) !\|f\|_{n}^{2}, \quad n=1,2, \ldots
$$


Furthermore, it is clear that we need only consider those functions

$$
f\left(t_{1}, \ldots, t_{k}, t\right)=\left(\prod_{i=1}^{k} 1_{I^{i}}\left(t_{i}\right)\right) 1_{I^{k+1}}(t)^{\mathrm{s}}, \quad I^{i}=\left(a_{i}, b_{i}\right], i=1, \ldots, k+1
$$

where $a_{1}<b_{1} \leqslant a_{1}<b_{2} \leqslant \ldots \leqslant a_{k}<b_{k} \leqslant a_{k+1}<b_{k+1}$. We proceed by induction. For $n=1, I_{0}(f(t))=f(t)$, so there is nothing to prove. Suppose that (5.5) is true for $n=k$, and consider the case $n=k+1$. Note that $n-1=k$ and

$$
\begin{aligned}
I_{k}(f(\cdot, t)) & =k ! \int_{0<t_{1}<\ldots<t_{k-1}<s<1} f\left(t_{1}, \ldots, t_{k-1}, s, t\right) \mathrm{d} M_{t_{1}} \ldots \mathrm{d} M_{t_{k-1}} \mathrm{~d} M_{s} \\
& =k\left\{\int_{0}^{t-} I_{k-1}\left(f(\cdot, s, t) 1_{[0, s)}^{\otimes k-1}\right) \mathrm{d} M_{s}+\int_{t+}^{\infty} I_{k-1}\left(f(\cdot, s, t) 1_{[0, s)}^{\otimes k-1}\right) \mathrm{d} M_{s}\right\} \\
& =V_{1}(t)+V_{2}(t) .
\end{aligned}
$$

Here we have used the fact that $f(\cdot, t, t)=0, \forall t \in T$, and $f(\cdot, s, t)=0$, for $s>1$. Since, for $\left(t_{1}, \ldots, t_{k-1}, s\right) \in C_{k}$ and $s<t$, we have

$$
f\left(t_{1}, \ldots, t_{k-1}, s, t\right)=\prod_{i=1}^{k-1} 1_{\left(a_{i}, b_{i}\right)}\left(t_{i}\right) 1_{\left(a_{k}, b_{k}\right)}(s) 1_{\left(a_{k-1}, b_{k+1}\right]}(t),
$$

the equality

$$
I_{k-1}\left(f(\cdot, s, t) 1_{[0, s)}^{\otimes k-1}\right)=I_{k-1}\left(\hat{f}(\cdot, s) 1_{[0, s)}^{\otimes k-1}(\cdot)\right) 1_{I^{k+1}}(t)=g_{k}(s) 1_{I^{k+1}}(t),
$$

holds, where $\hat{f}\left(t_{1}, \ldots, t_{k-1}, s\right):=\prod_{i=1}^{k-1} 1_{\left(a_{i}, b_{i}\right]}\left(t_{i}\right) 1_{\left(a_{k}, b_{k}\right]}(s), \quad g_{k}(s):=I_{k-1}\left(\hat{f}(\cdot, s) 1_{[0, s)}^{\otimes k-1}(\cdot)\right)$ and $I^{k+1}=\left(a_{k+1}, b_{k+1}\right]$. It is easily seen that $g_{k}(\cdot)$ is predictable, and so is $1_{I^{k+1}}(\cdot) \int_{0}^{*} g_{k}^{2}(s) \mathrm{d} s$. Noting that $\mathrm{E}[M, M]_{t}=t$, we have

$$
\begin{aligned}
\left\|V_{1}(\cdot)\right\|_{L^{2}(\mathrm{~d} \mu)}^{2} & =\int_{0}^{\infty} \mathrm{E} V_{1}(t)^{2} \mathrm{~d} t=k^{2} \int_{0}^{\infty} \mathrm{E}\left\{\int_{0}^{t-} 1_{I^{k+1}}(t) g_{k}(s) \mathrm{d} M_{s}\right\}^{2} \mathrm{~d} t \\
& =k^{2} \mathrm{E} \int_{0}^{\infty}\left\{\int_{0}^{t} 1_{I^{k+1}}(t) g_{k}^{2}(s) \mathrm{d} s\right\} \mathrm{d}[M, M]_{t} .
\end{aligned}
$$

In order similarly to determine $\left\|V_{2}\right\|_{L^{2}(\mathrm{~d} \mu)}^{2}$, let us define a stopping time $\tau_{t}:=$ inf $\left\{s \geqslant 0:[M, M]_{s}>t\right\} \wedge 1$. Then we have 


$$
\begin{aligned}
\left\|V_{2}(\cdot)\right\|_{L^{2}(\mathrm{~d} \mu)}^{2} & =k^{2} \mathrm{E} \int_{0}^{\infty}\left\{\int_{(t, \infty)} 1_{I^{k+1}}(t) g_{k}(s) \mathrm{d} M_{s}\right\}^{2} \mathrm{~d}[M, M]_{t} \\
& =k^{2} \mathrm{E} \int_{0}^{\infty} 1_{I^{k+1}}\left(\tau_{t}\right)\left\{\int_{\tau_{t}+}^{\infty} g_{k}(s) \mathrm{d} M_{s}\right\}^{2} \mathrm{~d} t \\
& =k^{2} \int_{0}^{\infty} \mathrm{E}\left\{\int_{\tau_{t}+}^{\infty} 1_{I^{k+1}}\left(\tau_{t}\right) g_{k}(s) \mathrm{d} M_{s}\right\}^{2} \mathrm{~d} t .
\end{aligned}
$$

Define, for fixed $t$, a process $G(t, s, \omega)=1_{I^{k+1}}\left(\tau_{t}(\omega)\right) g_{k}(s, \omega)$; then $G(t, \cdot, \cdot)$ is predictable for $s>\tau_{t}$. Thus

$$
E\left\{\int_{\tau_{t}+}^{\infty} 1_{I^{k+1}}\left(\tau_{t}\right) g_{k}(s) \mathrm{d} M_{s}\right\}^{2}=\mathrm{E} \int_{\tau_{t}+}^{\infty} 1_{I^{k+1}}\left(\tau_{t}\right) g_{k}^{2}(s) \mathrm{d} s
$$

Therefore the right-hand side of (5.9) becomes

$$
k^{2} \int_{0}^{\infty} \mathrm{E} \int_{\tau_{t}+}^{\infty} 1_{I^{k+1}}\left(\tau_{t}\right) g_{k}^{2}(s) \mathrm{d} s \mathrm{~d} t=k^{2} \mathrm{E} \int_{0}^{\infty}\left\{\int_{t+}^{\infty} 1_{I^{k+1}}(t) g_{k}^{2}(s) \mathrm{d} s\right\} \mathrm{d}[M, M]_{t} .
$$

Combining this with (5.8) and (5.9), and applying Fubini's theorem to $\mathrm{d} s \mathrm{~d}[M, M]_{t}$ (path by path), we have (recall (5.7) for the definition of $g_{k}$ ) that

$$
\begin{aligned}
\left\|V_{1}\right\|_{L^{2}(\mathrm{~d} \mu)}^{2}+\left\|V_{2}\right\|_{L^{2}(\mathrm{~d} \mu)}^{2} & =k^{2} \mathrm{E} \int_{0}^{\infty}\left\{\int_{0}^{\infty} 1_{I^{k+1}}(t) g_{k}^{2}(s) \mathrm{d} s\right\} \mathrm{d}[M, M]_{t} \\
& =k^{2} \mathrm{E} \int_{0}^{\infty} \int_{0}^{\infty} I_{k-1}^{2}\left(f(\cdot, s, t) 1_{[0, s)}^{\otimes k-1}(\cdot)\right) \mathrm{d}[M, M]_{t} \mathrm{~d} s .
\end{aligned}
$$

Moreover, by (5.8) and (5.9), we also see that

$$
\begin{aligned}
\left\|V_{1}\right\|_{L^{2}(\mathrm{~d} \mu)}^{2} & =k^{2} \mathrm{E} \int_{0}^{\infty} 1_{I^{k+1}}(t)\left\{\int_{0}^{t} g_{k}^{2}(s) \mathrm{d} s\right\} \mathrm{d} t \\
& \leqslant k^{2}(k-1) ! \int_{0}^{\infty} \int_{0}^{t}\left\|f(\cdot, s, t) 1_{[0, s)}^{\otimes k-1}(\cdot)\right\|_{k-1}^{2} \mathrm{~d} s \mathrm{~d} t \\
& \leqslant k k !\|f\|_{k+1}^{2}<\infty
\end{aligned}
$$

and, again by Fubini's theorem, that 


$$
\begin{aligned}
\left\|V_{2}\right\|_{L^{2}(\mathrm{~d} \mu)}^{2} & =k^{2} \mathrm{E} \int_{0}^{\infty}\left\{\int_{t+}^{\infty} 1_{I^{k+1}}(t) g_{k}^{2}(s) \mathrm{d} s\right\} \mathrm{d}[M, M]_{t} \\
& =k^{2} \mathrm{E} \int_{0}^{\infty}\left\{\int_{[0, s)} 1_{I^{k+1}}(t) g_{k}^{2}(s) \mathrm{d}[M, M]_{t}\right\} \mathrm{d} s \\
& =k^{2} \int_{0}^{\infty} \mathrm{E}\left\{\int_{0}^{\infty} I_{k-1}^{2}\left(f(\cdot, s, t) 1_{[0, s)}^{\otimes k-1}(\cdot)\right) 1_{[0, s)}(t) \mathrm{d}[M, M]_{t}\right\} \mathrm{d} s
\end{aligned}
$$

by the definition of $g_{k}$. Therefore, if we define for each fixed $s$ a function $h_{s}(\cdot, t)=$ $f(\cdot, s, t) 1_{[0, s)}^{\otimes k-1}(\cdot) 1_{[0, s)}(t)$, then $h_{s}(\cdot, t)$ is symmetric in the first $k-1$ variables. Using the induction hypothesis, we have

$$
\left(h_{s}, h_{s}\right)_{k}=\mathrm{E} \int_{0}^{\infty} I_{k-1}^{2}\left(f(\cdot, s, t) 1_{[0, s)}^{\otimes k-1}(\cdot)\right) 1_{[0, s)}(t) \mathrm{d}[M, M]_{t}=(k-1) !\left\|h_{s}\right\|_{k}^{2} .
$$

Combining (5.12) with (5.11), we obtain that

$$
\left\|V_{2}\right\|_{L^{2}(\mathrm{~d} \mu)}^{2}=k^{2}(k-1) ! \int_{0}^{\infty}\left\|h_{s}\right\|_{k}^{2} \mathrm{~d} s \leqslant k^{2}(k-1) !\|f\|_{k+1}^{2}<\infty .
$$

In other words, we have shown that $V_{1}, V_{2} \in L^{2}(\mathrm{~d} \mu)$, whence $V_{1} V_{2} \in L^{1}(\mathrm{~d} \mu)$. We now show that

$$
\mathrm{E} \int_{0}^{\infty} V_{1}(t) V_{2}(t) \mathrm{d}[M, M]_{t}=0
$$

In fact, using Lebesgue's change of time lemma (cf., for example, Dellacherie 1972, p. 91),

$$
\infty>\mathrm{E} \int_{0}^{\infty}\left|V_{1}(t) V_{2}(t)\right| \mathrm{d}[M, M]_{t}=E \int_{0}^{\infty}\left|V_{1}\left(\tau_{t}\right) V_{2}\left(\tau_{t}\right)\right| \mathrm{d} t=\int_{0}^{\infty} \mathrm{E}\left|V_{1}\left(\tau_{t}\right) V_{2}\left(\tau_{t}\right)\right| \mathrm{d} t,
$$

we see that for almost every $t \in[0,1], E\left|V_{1}\left(\tau_{t}\right) V_{2}\left(\tau_{t}\right)\right|<\infty$. Hence

$$
\begin{aligned}
\mathrm{E}\left\{V_{1}\left(\tau_{t}\right) V_{2}\left(\tau_{t}\right)\right\} & =\mathrm{E}\left\{V_{1}\left(\tau_{t}\right) \mathrm{E}\left\{V_{2}\left(\tau_{t}\right) \mid \mathscr{F}_{\tau_{t}}\right\}\right\} \\
& =\mathrm{E}\left\{V_{1}\left(\tau_{t}\right) E\left\{\int_{\tau_{t}+}^{\infty} 1_{I^{k+1}}\left(\tau_{t}\right) g_{k}(s) \mathrm{d} M_{s} \mid \mathscr{F}_{\tau_{t}}\right\}\right\} .
\end{aligned}
$$

Since $I^{k+1}\left(\tau_{t}\right) g_{k}(s)$ is predictable on $s>\tau_{t}$ for almost all $t$, we have $\mathrm{E}\left\{V_{1}\left(\tau_{t}\right) V_{2}\left(\tau_{t}\right)\right\}=0$ for almost all $t$, whence

$$
\mathrm{E} \int_{0}^{\infty} V_{1}(t) V_{2}(t) \mathrm{d}[M, M]_{t}=\int_{0}^{\infty} \mathrm{E}\left\{V_{1}\left(\tau_{t}\right) V_{2}\left(\tau_{t}\right)\right\} \mathrm{d} t=0,
$$

proving (5.14). Now by (5.6), (5.10) and (5.14) we obtain 


$$
\begin{aligned}
\left\|I_{k}(f(\cdot, \cdot))\right\|_{L^{2}(\mathrm{~d} \mu)}^{2} & =\left\|V_{1}(\cdot)+V_{2}(\cdot)\right\|_{L^{2}(\mathrm{~d} \mu)}^{2}=\left\|V_{1}\right\|_{L^{2}(\mathrm{~d} \mu)}^{2}+\left\|V_{2}\right\|_{L^{2}(\mathrm{~d} \mu)}^{2} \\
& =k^{2} \int_{0}^{\infty} \mathrm{E}\left\{\int_{0}^{\infty} I_{k-1}^{2}\left(f(\cdot, s, t) 1_{[0, s)}^{\otimes k-1}(\cdot)\right) \mathrm{d}[M, M]_{t}\right\} \mathrm{d} s:=k^{2} J .
\end{aligned}
$$

Using the induction hypothesis again, we see that

$$
\mathrm{E}\left\{\int_{0}^{\infty} I_{k-1}^{2}\left(f(\cdot, s, t) 1_{[0, s)}^{\otimes k-1}(\cdot)\right) \mathrm{d}[M, M]_{t}\right\}=(k-1) !\left\|f(\cdot, s, \cdot) 1_{[0, s)}^{\otimes k-1}(\cdot)\right\|_{k}^{2} .
$$

Thus

$$
\begin{aligned}
J & =\int_{0}^{\infty}(k-1) !\left\|f(\cdot, s, \cdot) 1_{[0, s)}^{\otimes k-1}(\cdot)\right\|_{k}^{2} \mathrm{~d} s \\
& =(k-1) ! \int_{0}^{\infty}\left\{\int_{0}^{\infty}\left[\int_{0}^{s} \ldots \int_{0}^{s}\left|f\left(t_{1}, \ldots, t_{k-1}, s, t\right)\right|^{2} \mathrm{~d} t_{1}, \ldots \mathrm{d} t_{k-1}\right] \mathrm{d} t\right\} \mathrm{d} s \\
& =(k-1) !(k-1) ! \int_{0}^{\infty}\left\{\int_{0<t_{1}<\ldots<t_{k-1}<s<\infty}\left|f\left(t_{1}, \ldots, t_{k-1}, s, t\right)\right|^{2} \mathrm{~d} t_{1} \ldots \mathrm{d} t_{k-1} \mathrm{~d} s\right\} \mathrm{d} t \\
& =((k-1) !)^{2} \cdot \frac{1}{k !} \int_{0}^{\infty}\left\{\int_{T^{k}}\left|f\left(t_{1}, \ldots, t_{k-1}, s, t\right)\right|^{2} \mathrm{~d} t_{1} \ldots \mathrm{d} t_{k-1} \mathrm{~d} s\right\} \mathrm{d} t \\
& =\frac{((k-1) !)^{2}}{k !}\|f\|_{k+1}^{2} .
\end{aligned}
$$

Therefore

$$
\left\|I_{k}(f(\cdot, \cdot))\right\|_{L^{2}(\mathrm{~d} \mu)}^{2}=k^{2} J=k^{2} \frac{((k-1) !)^{2}}{k !}\|f\|_{k+1}^{2}=k !\|f\|_{k+1}^{2},
$$

and (5.5) is proved.

Case $2(n \neq m)$. The proof is very similar, and we give only a sketch. We shall again use induction, but this time on $k=n \wedge m$. Assume that $k=1$. Let $n=1$ and $m \geqslant 2$. Then notice that $I_{0}(f(\cdot, t))=f(t)$; we have

$$
(f, g)_{1, m}=\mathrm{E} \int_{0}^{\infty} I_{0}(f(\cdot, t)) I_{m-1}(g(\cdot, t)) \mathrm{d}[M, M]_{t}=\mathrm{E} \int_{0}^{\infty} I_{m-1}(g(\cdot, t)) f(t) \mathrm{d}[M, M]_{t} .
$$

Since $g \in S_{m}$ and $f \in S_{1}$, we have by Lemma 5.1 that

$$
m \int_{0}^{\infty} I_{m-1}(g(\cdot, t)) f(t) \mathrm{d}[M, M]_{t}=I_{m}(g) I_{1}(f)-I_{m+1}(g \otimes h) .
$$

Therefore $(f, g)_{1, m}=m^{-1}\left\{\mathrm{E}\left\{I_{m}(g) I_{1}(f)\right\}-\mathrm{E} I_{m+1}(g \otimes f)\right\}=0$.

Next assume that the conclusion is true for $n \wedge m=k$, and consider the case when $n=k$ and $m \geqslant k+1$. Write

$$
V_{1}(t)=(n-1) \int_{0}^{t-} 1_{I^{n+1}}(t) \hat{f}_{n}(s) \mathrm{d} M_{s}, \quad V_{2}(t)=(n-1) \int_{t+}^{\infty} 1_{I^{n+1}}(t) \hat{f}_{n}(s) \mathrm{d} M_{s},
$$




$$
U_{1}(t)=(m-1) \int_{0}^{t-} 1_{I^{m+1}}(t) \hat{g}_{m}(s) \mathrm{d} M_{s}, \quad U_{2}(t)=(m-1) \int_{t+}^{\infty} 1_{I^{m+1}}(t) \hat{g}_{m}(s) \mathrm{d} M_{s},
$$

where

$$
\hat{f}_{n}(s)=I_{n-2}\left(\prod_{i=1}^{n-1} 1_{I^{i}}\left(t_{i}\right) 1_{I^{n}}(s) 1_{[0, s)}^{\otimes n-2}\right), \quad \hat{g}_{n}(s)=I_{m-2}\left(\prod_{j=1}^{m-1} 1_{I^{j}}\left(t_{j}\right) 1_{I^{m}}(s) 1_{[0, s)}^{\otimes m-2}\right) .
$$

Then it is easily seen as before that

$$
\begin{aligned}
& \mathrm{E} \int_{0}^{\infty} I_{n-1}(f(\cdot, t)) I_{m-1}(g(\cdot, t)) \mathrm{d}[M, M]_{t} \\
& \quad=\mathrm{E} \int_{0}^{\infty}\left(V_{1}(t)+V_{2}(t)\right)\left(U_{1}(t)+U_{2}(t)\right) \mathrm{d}[M, M]_{t} \\
& \quad=\mathrm{E} \int_{0}^{\infty}\left[V_{1}(t) U_{1}(t)+V_{1}(t) U_{2}(t)+V_{2}(t) U_{1}(t)+V_{2}(t) U_{2}(t)\right] \mathrm{d}[M, M]_{t} .
\end{aligned}
$$

Using the same argument as for (5.14), we have

$$
\mathrm{E} \int_{0}^{\infty} V_{1}(t) U_{2}(t) \mathrm{d}[M, M]_{t}=\mathrm{E} \int_{0}^{\infty} V_{2}(t) U_{1}(t) \mathrm{d}[M, M]_{t}=0 .
$$

By the predictability of $V_{1}$ and $U_{1}$, we have

$$
\begin{aligned}
\mathrm{E} \int_{0}^{\infty} V_{1}(t) U_{2}(t) \mathrm{d}[M, M]_{t} & =\mathrm{E} \int_{0}^{\infty} V_{1}(t) U_{1}(t) \mathrm{d} t=\int_{0}^{\infty} \mathrm{E}\left\{V_{1}(t) U_{1}(t)\right\} \mathrm{d} t \\
& =(n-1)(m-1) \int_{0}^{\infty} 1_{I^{n+1}}(t) 1_{I^{m+1}}(t) \mathrm{E}\left\{\int_{0}^{t-} \hat{f}(s) \hat{g}(s) \mathrm{d} s\right\} \mathrm{d} t=0 .
\end{aligned}
$$

Finally, note that

$$
\mathrm{E} \int_{0}^{\infty} V_{2}(t) U_{2}(t) \mathrm{d}[M, M]_{t}=\int_{0}^{\infty} \mathrm{E}\left\{V_{1}\left(\tau_{t}\right) U_{2}\left(\tau_{t}\right)\right\} \mathrm{d} t
$$

and

$$
V_{2}\left(\tau_{t}\right)=\int_{0}^{\infty} 1_{I^{n+1}}\left(\tau_{t}\right) \hat{f}(s) 1_{\left\{s>\tau_{t}\right\}} \mathrm{d} M_{s}, \quad U_{2}\left(\tau_{t}\right)=\int_{0}^{\infty} 1_{I^{m+1}}\left(\tau_{t}\right) \hat{g}(s) 1_{\left\{s>\tau_{t}\right\}} \mathrm{d} M_{s} .
$$

Since both integrands are predictable, we have

$$
\begin{aligned}
\int_{0}^{\infty} \mathrm{E}\left\{V_{2}\left(\tau_{t}\right) U_{2}\left(\tau_{t}\right)\right\} \mathrm{d} t & =\int_{0}^{\infty} \mathrm{E}\left\{\int_{0}^{\infty} 1_{I^{n+1}}\left(\tau_{t}\right) \hat{f}(s) 1_{I^{m+1}}\left(\tau_{t}\right) \hat{g}(s) 1_{\left\{s>\tau_{t}\right\}} \mathrm{d} s\right\} \mathrm{d} t \\
& =\mathrm{E} \int_{0}^{\infty} \int_{0}^{\infty} 1_{I^{n+1}}(t) \hat{f}(s) 1_{I^{m+1}}(t) \hat{g}(s) 1_{\{s>t\}} \mathrm{d} s \mathrm{~d}[M, M]_{t} .
\end{aligned}
$$


Combining the equalities $f(\cdot, t, t)=g(\cdot, t, t)=0$ and

$$
\mathrm{E} \int_{0}^{\infty} \int_{0}^{\infty} 1_{I^{n+1}}(t) \hat{f}(s) 1_{I^{m+1}}(t) \hat{g}(s) 1_{\{s<t\}} \mathrm{d} s \mathrm{~d}[M, M]_{t}=\mathrm{E}\left\{V_{1}(t) U_{1}(t)\right\}=0
$$

with the induction hypothesis, we conclude that

$$
\mathrm{E} \int_{0}^{\infty} 1_{I^{n+1}}(t) \hat{f}(s) 1_{I^{m+1}}(t) \hat{g}(s) \mathrm{d}[M, M]_{t}=0, \quad \text { a.e. } s .
$$

Finally, we see from (5.16), (5.17) and (5.18) that

$$
\begin{aligned}
\mathrm{E} \int_{0}^{\infty} V_{2}(t) U_{2}(t) \mathrm{d}[M, M]_{t} & =\mathrm{E} \int_{0}^{\infty} \int_{0}^{\infty} 1_{I^{n+1}}\left(\tau_{t}\right) \hat{f}(s) 1_{I^{m+1}}\left(\tau_{t}\right) \hat{g}(s) \mathrm{d} s \mathrm{~d}[M, M]_{t} \\
& =\int_{0}^{\infty} \mathrm{E}\left\{\int_{0}^{\infty} 1_{I^{n+1}}(t) \hat{f}(s) 1_{I^{m+1}}(t) \hat{g}(s) \mathrm{d}[M, M]_{t}\right\} \mathrm{d} s=0 .
\end{aligned}
$$

The proof is thus complete.

Lemma 5.2 indicates an interesting fact: that if $f_{n} \in S_{n+1}$, then $I_{n}\left(f_{n}(\cdot, t)\right) \in$ $L^{2}(\mathrm{~d} \mu) \cap L^{2}(\mathrm{~d} \lambda)$, where $\mathrm{d} \lambda=\mathrm{d} t \times \mathrm{d} P$, and the sequence $\left\{I_{n}\left(f_{n}(\cdot, t)\right)\right\}$ is orthogonal in both $L^{2}(\mathrm{~d} \mu)$ and $L^{2}(\mathrm{~d} \lambda)$, such that

$$
\left\|I_{n}(f(\cdot, \cdot))\right\|_{L^{2}(\mathrm{~d} \mu)}^{2}=\left\|I_{n}(f(\cdot, \cdot))\right\|_{L^{2}(\mathrm{~d} \lambda)}^{2}=n !\|f\|_{n+1}^{2} .
$$

Therefore, since $S_{n+1}$ is dense in $L_{s}^{2}\left(T^{n+1}\right)$, for any $f \in L_{s}^{2}\left(T^{n+1}\right)$, we can take a sequence $\left\{f^{k}\right\}$ converging to $f$ in $L^{2}\left(T^{n+1}\right)$, such that $f^{k} \in S_{n+1}, k=1,2, \ldots$. Hence we can define two limit processes,

$$
\begin{array}{ll}
F(t)=\lim _{k \rightarrow \infty} I_{n}\left(f^{k}(\cdot, t)\right) & \text { in } L^{2}(\mathrm{~d} \mu), \\
G(t)=\lim _{k \rightarrow \infty} I_{n}\left(f^{k}(\cdot, t)\right) & \text { in } L^{2}(\mathrm{~d} \lambda),
\end{array}
$$

such that $\|F\|_{L^{2}(\mathrm{~d} \mu)}^{2}=\|G\|_{L^{2}(\mathrm{~d} \lambda)}^{2}=n !\|f\|_{n+1}^{2}$. We now define a new measure on $[0,1] \times \Omega$ by

$$
v([0, t) \times B)=\frac{\lambda([0, t) \times B)+\mu([0, t) \times B)}{2}, \quad t \in[0,1], B \in \mathscr{F},
$$

and

$$
I_{n}^{*}(f(\cdot, t)):=\lim _{k \rightarrow \infty} I_{n}\left(f^{(k)}(\cdot, t)\right) \quad \text { in } L^{2}(\mathrm{~d} v) .
$$

Then $I_{n}^{*}(f(\cdot, t))$ is well defined and satisfies

$$
I_{n}^{*}(f(\cdot, t))= \begin{cases}F(t) & \text { a.e. } \mathrm{d} \mu \\ G(t) & \text { a.e. } \mathrm{d} \lambda\end{cases}
$$


We now turn our attention to the integration by parts formula. Recall that in the Brownian case we have, for any $F, G \in \mathbb{D}_{1,2}$ and $h \in H:=L^{2}(T)$,

$$
\mathrm{E}\left\{G\langle D F, h\rangle_{T}+F\langle D G, h\rangle_{T}\right\}=\mathrm{E}\left\{F G \int_{0}^{1} h(t) \mathrm{d} W_{t}\right\},
$$

where $W$ is the standard Wiener process. We will show an analogue of (5.21) in our case. First we give a theorem inspired by a result in Russo and Vallois (1994). (Note that the result of Russo and Vallois is slightly incorrect, since $I_{n-1}$ replaces $I_{n-1}^{*}$.)

Theorem 5.3. Suppose that $n \geqslant 1, f \in L_{s}^{2}\left(T^{n}\right)$ and $h \in L^{2}(T)$; then

$$
I_{n}(f) I_{1}(h)=I_{n+1}(f \otimes h)+n \int_{0}^{\infty} I_{n-1}^{*}(f(\cdot, t)) h(t) \mathrm{d}[M, M]_{t} .
$$

Proof. We follow the idea of Russo and Vallois. First assume that $f \in S_{n}$ and $h \in L^{2}(T)$. By Lemma 5.1 we have

$$
I_{n}(f) I_{1}(h)=I_{n+1}(f \otimes h)+n \int_{0}^{\infty} I_{n-1}(f(\cdot, t)) h(t) \mathrm{d}[M, M]_{t} .
$$

Now letting $f \in L_{s}^{2}\left(T^{n}\right)$ and $h \in L^{2}(T)$, we can find a sequence $\left\{f^{k}\right\} \subset S_{n}$ such that $\lim _{k \rightarrow \infty} f^{k}=f$ in $L_{s}^{2}\left(T^{n}\right)$. By Lemma 5.2 and the discussion following it, we see that

$$
\begin{array}{ll}
\lim _{k \rightarrow \infty} I_{n}\left(f^{k}\right)=I_{n}(f) & \text { in } L^{2}(\Omega), \\
\lim _{k \rightarrow \infty} I_{n+1}\left(f^{k} \otimes h\right)=I_{n+1}(f \otimes h) & \text { in } L^{2}(\Omega), \\
\lim _{k \rightarrow \infty} I_{n-1}\left(f^{k}(\cdot, \cdot)\right)=I_{n-1}^{*}(f(\cdot, \cdot)) & \text { in } L^{2}(\mathrm{~d} \mu) .
\end{array}
$$

Furthermore, noting that $\mathrm{E} \int_{0}^{1}|h(t)|^{2} \mathrm{~d}[M, M]_{t}=\int_{0}^{1}|h(t)|^{2} \mathrm{~d} t=\|h\|_{1}^{2}$, we have by the Cauchy-Schwarz inequality that

$$
\begin{aligned}
& \mathrm{E}\left|\int_{0}^{1} I_{n-1}\left(f^{k}(\cdot, t)\right) h(t) \mathrm{d}[M, M]_{t}-\int_{0}^{1} I_{n-1}^{*}(f(\cdot, t)) h(t) \mathrm{d}[M, M]_{t}\right| \\
& \quad \leqslant \mathrm{E} \int_{0}^{1}\left|I_{n-1}\left(f^{k}(\cdot, t)\right)-I_{n-1}^{*}(f(\cdot, t))\right||h(t)| \mathrm{d}[M, M]_{t} \\
& \quad \leqslant\left\|I_{n-1}\left(f^{k}(\cdot, \cdot)\right)-I_{n-1}^{*}(f(\cdot, \cdot))\right\|_{L^{2}(\mathrm{~d} \mu)}\|h\|_{1} \rightarrow 0 .
\end{aligned}
$$

Therefore, taking limits on both sides of (5.23) (replacing $f$ by $f^{k}$ ) in $L^{1}(\Omega)$, we obtain (5.22), proving the theorem. 
Let us now define an operator $D^{*}: L^{2}(\Omega) \mapsto L^{2}(\mathrm{~d} v)$ by

$$
D_{t}^{*} I_{n}(f)=n I_{n-1}^{*}(f(\cdot, t)), \quad \forall f \in L_{s}^{2}\left(T^{n}\right) ;
$$

and for $F=\sum_{n=0}^{\infty} I_{n}\left(f_{n}\right)$, such that $\sum_{n=0}^{\infty} n n !\left\|f_{n}\right\|_{n}^{2}<\infty$, we define

$$
D_{t}^{*} F=\sum_{n=0}^{\infty} n I_{n-1}^{*}\left(f_{n}(\cdot, t)\right), \quad t \in[0,1] .
$$

We have the following analogue of (5.21).

Theorem 5.4. Suppose that $G \in L^{2}(\Omega)$ has the chaos expansion $G=\sum_{n=0}^{\infty} I_{n}\left(g_{n}\right)$, where $g_{n} \in L_{s}^{2}\left(T^{n}\right)$ and $\sum_{n=0}^{\infty} \sqrt{n n !}\left\|g_{n}\right\|_{n}<\infty$. Then for any $F \in \mathbb{D}_{1,2}$ and $h \in L^{2}(T)$,

$$
\mathrm{E}\left\{G\langle D F, h\rangle_{T}\right\}=\mathrm{E}\left\{F\left(G \int_{0}^{1} h(t) \mathrm{d} M_{t}-\int_{0}^{1} D_{t}^{*} G h(t) \mathrm{d}[M, M]_{t}\right)\right\} .
$$

Proof. Let $n \geqslant 1$ be fixed. Since $g_{n} \in L_{s}^{2}\left(T^{n}\right)$, we have by Theorem 5.2 that

$$
I_{n}\left(g_{n}\right) I_{1}(h)=I_{n+1}\left(g_{n} \otimes h\right)+n \int_{0}^{1} I_{n-1}^{*}\left(g_{n}(\cdot, t)\right) h(t) \mathrm{d}[M, M]_{t} .
$$

For any $k, m \in \mathbb{N}$, we have by the Cauchy-Schwarz inequality that

$$
\begin{aligned}
& \mathrm{E} \int_{0}^{1}\left|\sum_{n=1}^{m} n I_{n-1}^{*}\left(g_{n}(\cdot, t)\right)-\sum_{n=1}^{k} n I_{n-1}^{*}\left(g_{n}(\cdot, t)\right)\right||h(t)| \mathrm{d}[M, M]_{t} \\
& \quad \leqslant \sum_{n=k+1}^{m} \mathrm{E} \int_{0}^{1}\left|n I_{n-1}^{*}\left(g_{n}(\cdot, t)\right)\right||h(t)| \mathrm{d}[M, M]_{t} \\
& \quad \leqslant \sum_{n=k+1}^{m} n \sqrt{(n-1) !}\left\|g_{n}\right\|_{n}\|h\|_{1} \rightarrow 0, \quad \text { as } k, m \rightarrow \infty
\end{aligned}
$$

by assumption (note that $n \sqrt{(n-1) !}=\sqrt{n n !}$ ). Therefore, $\sum_{n=0}^{k} n I_{n-1}^{*}\left(g_{n}(\cdot, \cdot)\right) h(\cdot) \rightarrow$ $D^{*} G h(\cdot)$, as $k \rightarrow \infty$, in $L^{1}(\mathrm{~d} \mu)$. Consequently,

$$
\begin{aligned}
G \int_{0}^{1} h(t) \mathrm{d} M_{t} & =\sum_{n=0}^{\infty} I_{n}\left(g_{n}\right) I_{1}(h) \\
& =\sum_{n=0}^{\infty} I_{n+1}\left(g_{n} \otimes h\right)+\int_{0}^{1} \sum_{n=1}^{\infty} n I_{n-1}^{*}\left(g_{n}(\cdot, t)\right) h(t) \mathrm{d}[M, M]_{t} \\
& =\sum_{n=0}^{\infty} I_{n+1}\left(g_{n} \otimes h\right)+\int_{0}^{1} D_{t}^{*} G h(t) \mathrm{d}[M, M]_{t} \quad \text { in } L^{1}(\Omega),
\end{aligned}
$$


or equivalently,

$$
\sum_{n=0}^{\infty} I_{n+1}(g \otimes h)=G \int_{0}^{1} h(t) \mathrm{d} M_{t}-\int_{0}^{1} D_{t}^{*} G h(t) \mathrm{d}[M, M]_{t} .
$$

Note that since the left-hand side of (5.26) is in $L^{2}(\Omega)$, so also is the right-hand side. Thus for any $F \in \mathbb{D}_{1,2} \subset L^{2}(\Omega)$, we have

$$
F\left\{\sum_{n=0}^{\infty} I_{n+1}(g \otimes h)\right\}=F\left\{G \int_{0}^{1} h(t) \mathrm{d} M_{t}-\int_{0}^{1} D_{t}^{*} G h(t) \mathrm{d}[M, M]_{t}\right\},
$$

and the equality holds in $L^{1}(\Omega)$. If we write $F=\sum_{k=0}^{\infty} I_{k}\left(f_{k}\right)$ for its chaos expansion, then $\sum_{k=0}^{\infty} k k !\left\|f_{k}\right\|_{k}^{2}<\infty$ since $F \in \mathbb{D}_{1,2}$, and therefore

$$
\begin{aligned}
\mathrm{E}\left\{F \sum_{n=0}^{\infty} I_{n+1}\left(g_{n} \otimes h\right)\right\} & =\mathrm{E}\left\{\sum_{k} I_{k}\left(f_{k}\right) \cdot \sum_{n} I_{n+1}\left(g_{n} \otimes h\right)\right\} \\
& =\sum_{k} \mathrm{E}\left\{I_{k}\left(f_{k}\right) I_{k}\left(g_{k-1} \otimes h\right)\right\} \\
& =\sum_{k} k !\left\langle f_{k}, g_{k-1} \otimes h\right\rangle_{T^{k}} \\
& =\sum_{k}(k-1) ! \int_{0}^{1}\left\langle k f_{k}(\cdot, t), g_{k-1}\right\rangle_{T^{k-1}} h(t) \mathrm{d} t \\
& =\int_{0}^{1} \sum_{k} \mathrm{E}\left(k I_{k-1}\left(f_{k}(\cdot, t)\right), I_{k-1}\left(g_{k-1}\right)\right) h(t) \mathrm{d} t \\
& =\int_{0}^{1} \mathrm{E}\left\{\sum_{k} k I_{k-1}\left(f_{k}(\cdot, t)\right) \cdot \sum_{n} I_{n}\left(g_{n}\right)\right\} h(t) \mathrm{d} t \\
& =\mathrm{E} \int_{0}^{1} D_{t} F G h(t) \mathrm{d} t=\mathrm{E}\left\{G\left\langle D_{t} F, h\right\rangle_{T}\right\} .
\end{aligned}
$$

Taking expectations on both sides of (5.27), and replacing its left-hand side by (5.28), we obtain (5.25). The theorem is proved.

Corollary 5.5. Suppose that $F, G \in L^{2}(\Omega)$ with $F=\sum_{n} I_{n}\left(f_{n}\right), G=\sum_{k} I_{k}\left(g_{k}\right)$, such that

$$
\sum_{n} \sqrt{n n !}\left\|f_{n}\right\|_{n}<\infty, \quad \sum_{k} \sqrt{k k !}\left\|g_{k}\right\|_{k}<\infty .
$$

Assume that $h \in L^{2}(T)$ and $\mathrm{E}\left|F G \int_{0}^{1} h(t) \mathrm{d} M_{t}\right|<\infty$; then 


$$
\mathrm{E}\left\{F G \int_{0}^{1} h(t) \mathrm{d} M_{t}\right\}=\int_{[0,1] \times \Omega}\left\{F D_{t}^{*} G+G D_{t}^{*} F\right\} h(t) \mathrm{d} v .
$$

In particular, if $M$ is a Brownian motion, then (5.30) becomes (5.21).

Proof. First, note that if $F=\sum_{n} I_{n}\left(f_{n}\right)$ and (5.29) holds, then $F \in \mathbb{D}_{1,2}$. Indeed, for $n$ large enough, one has $n n !\left\|f_{n}\right\|_{n}^{2} \leqslant \sqrt{n n !}\left\|f_{n}\right\|_{n}<1$. Thus $\sum_{n} n n !\left\|f_{n}\right\|_{n}^{2}<\infty$ and $F \in \mathbb{D}_{1,2}$. In other words, under condition (5.29), $F, G \in \mathbb{D}_{1,2}$.

We can now apply Theorem 5.4 twice with the positions of $F$ and $G$ switched, and add the resulting equalities together to get

$$
\mathrm{E}\left\{G\langle D F, h\rangle_{T}+F\langle D G, h\rangle_{T}\right\}=\mathrm{E}\left\{2 F G \int_{0}^{1} h(t) \mathrm{d} M_{t}-\int_{0}^{1}\left[F D_{t}^{*} G+G D_{t}^{*} F\right] h(t) \mathrm{d}[M, M]_{t}\right\} .
$$

Since $F G \int_{0}^{1} h(t) \mathrm{d} M_{t} \in L^{1}(\Omega)$ by assumption, we obtain from (5.31) that

$$
\int_{0}^{1}\left[F D_{t}^{*} G+G D_{t}^{*} F\right] h(t) \mathrm{d}[M, M]_{t} \in L^{1}(\Omega) .
$$

Furthermore, noticing that $D_{.}^{*} F=D . F ; D^{*} G=D . G$, a.e. $\mathrm{d} t \times \mathrm{d} P$, we deduce from (5.31) that

$$
\begin{aligned}
\mathrm{E}\left\{F G \int_{0}^{1} h(t) \mathrm{d} M_{t}\right\} & =\frac{1}{2} \mathrm{E}\left\{\int_{0}^{1}\left[F D_{t}^{*} G+G D_{t}^{*} F\right] h(t)\left(\mathrm{d}[M, M]_{t}+\mathrm{d} t\right)\right\} \\
& =\int_{[0,1] \times \Omega}\left[F D_{t}^{*} G+G D_{t}^{*} F\right] h(t) \mathrm{d} v,
\end{aligned}
$$

proving the first assertion. In the case when $M$ is a Brownian motion, we have $[M, M]_{t}=\langle M, M\rangle_{t}=t$. Hence $\mathrm{d} v=\mathrm{d} t \times \mathrm{d} P, \quad D_{t}^{*} F=D_{t} F$ and $D_{t}^{*} G=D_{t} G$. Consequently,

$$
\begin{aligned}
\int_{[0,1] \times \Omega}\left[F D_{t}^{*} G+G D_{t}^{*} F\right] h(t) \mathrm{d} v & =\mathrm{E}\left\{\int_{0}^{1}\left[F D_{t} G+G D_{t} F\right] h(t) \mathrm{d} t\right\} \\
& =\mathrm{E}\left\{F\langle D G, h\rangle_{T}+G\langle D F, h\rangle_{T}\right\} .
\end{aligned}
$$

The proof is thus complete.

We remark that in Theorem 5.4 and Corollary 5.5 no anticipating integral is involved. To conclude this section we shall present another formula that does involve the anticipating integrals. In the Brownian case, this formula is nothing but the definition of an adjoint relation (see (4.3)). Our formula involves the measure $\mathrm{d}[M, M]_{t}$, therefore the anticipating integral for a special class of processes involving $I_{n}^{*}$ has to be considered. 
Let us consider a class of processes of the form $u_{t}=\sum_{m=0}^{\infty} I_{m}^{*}\left(f_{m}(\cdot, t)\right)$, where $f_{m} \in L_{s}^{2}\left(T^{m+1}\right)$ and the convergence of the series is in the sense of $L^{2}(\mathrm{~d} v)$. We say that a process of this kind is of class $\mathscr{B}^{*}$, if

$$
\sum_{m=0}^{\infty}(m+1) !\left\|f_{m}\right\|_{L^{2}\left(T^{m+1}\right)}^{2}<\infty
$$

Denote by $\operatorname{Dom}\left(\delta^{*}\right)$ the set of all processes of class $\mathscr{L}^{*}$. We consider the anticipating integral for processes in $\operatorname{Dom}\left(\delta^{*}\right)$. First note that for each $n$ we can find a sequence $\left\{f_{m}^{k}\right\} \subset S_{m+1}$ such that $\lim _{k \rightarrow \infty} f_{m}^{k}=f_{m}$ in $L^{2}\left(T^{m+1}\right)$. Define, for every pair of integers $N$ and $k$, a process

$$
u_{t}^{N, k}=\sum_{m=0}^{N} I_{m}^{*}\left(f_{m}^{k}(\cdot, t)\right)=\sum_{m=0}^{N} I_{m}\left(f_{m}^{k}(\cdot, t)\right), \quad \forall t \in[0,1]
$$

Then by definition (5.20), we see that $\lim _{N \rightarrow \infty} \lim _{k \rightarrow \infty} u^{N, k}=u$., in $L^{2}(\mathrm{~d} v)$. On the other hand, for fixed $N$ and $k$, we have $u^{N, k} \in \operatorname{Dom}(\delta)$ and $\delta\left(u^{N, k}\right)=\sum_{m=0}^{N} I_{m+1}\left(f_{m}^{k}\right)$ by definition (note that the $f_{m}^{k}$ are symmetric); thus if $u$ is of class $\mathscr{L}^{*}$, then

$$
\lim _{N \rightarrow \infty} \lim _{k \rightarrow \infty} \delta\left(u^{N, k}\right)=\lim _{N \rightarrow \infty} \lim _{k \rightarrow \infty} \sum_{m=0}^{N} I_{m+1}\left(f_{m}^{k}\right)=\sum_{m=0}^{\infty} I_{m+1}\left(f_{m}\right) \quad \text { in } L^{2}(\Omega) .
$$

We define the anticipating integral of $u \in \mathscr{D}^{*}$ to be

$$
\delta^{*}(u):=\sum_{m=0}^{\infty} I_{m+1}\left(f_{m}\right)
$$

Clearly, the value of $\delta^{*}(u)$ is independent of the choice of the approximating sequence, and it coincides with $\delta(u)$ if, in the expansion of $u$, all $f_{m} \in S_{m+1}$. Using the notation $\delta^{*}$ and $D^{*}$, we now give a new integration by parts formula.

Theorem 5.6. Suppose that $u \in \operatorname{Dom}\left(\delta^{*}\right)$ and $G \in \mathbb{D}_{1,2}$. Then

$$
\mathrm{E}\left\{\delta^{*}(u) G\right\}=\mathrm{E}\left\{\int_{0}^{1} u_{t}\left(D_{t}^{*} G\right) \mathrm{d}[M, M]_{t}\right\}=\mathrm{E}\left\{\int_{0}^{1} u_{t}\left(D_{t}^{*} G\right) \mathrm{d} t\right\} .
$$

Consequently,

$$
\mathrm{E}\left\{\delta^{*}(u) G\right\}=\int_{[0,1] \times \Omega} u_{t}(\omega)\left(D_{t}^{*} G\right)(\omega) v(\mathrm{~d} t \times \mathrm{d} \omega) .
$$


Proof. First we note that (5.34) follows from (5.33) by definition (5.19); therefore we need only show (5.33). To begin with, let us choose for each $n$ and $m$ two sequences $\left\{f_{m}^{k}\right\}_{k=1}^{\infty} \subset S_{m+1}$, and $\left\{g_{n}^{\prime}\right\} \subset S_{n}$, such that $\lim _{k \rightarrow \infty} f_{m}^{k}=f_{m}$ in $L^{2}\left(T^{m+1}\right)$ and $\lim _{\ell \rightarrow \infty} g_{n}^{\ell}=g_{n}$ in $L^{2}\left(T^{n}\right)$. Now, for given integers $N, k$ and $\ell$, we define

$$
u_{t}^{N, k}=\sum_{m=0}^{N} I_{m}\left(f_{m}^{k}(\cdot, t)\right), \quad G^{N, \ell}=\sum_{n=0}^{N} I_{n}\left(g_{n}^{\ell}\right) .
$$

It is easily seen by definitions (5.32) (for $\left.\delta^{*}\right),(5.24)$ (for $D^{*}$ ) and (5.20) (for $I_{n}^{*}$ ) that

$$
\begin{aligned}
\lim _{N \rightarrow \infty} \lim _{k \rightarrow \infty} u^{N, k} & =\lim _{N \rightarrow \infty} \sum_{m=0}^{N} I_{m}^{*}\left(f_{m}(\cdot, \cdot)\right)=u \quad \text { in } L^{2}(\mathrm{~d} v), \\
\lim _{N \rightarrow \infty} \lim _{k \rightarrow \infty} \delta\left(u^{N, k}\right) & =\delta^{*}(u) \quad \text { in } L^{2}(\Omega), \\
\lim _{N \rightarrow \infty} \lim _{\ell \rightarrow \infty} D \cdot G^{N, \ell} & =\lim _{N \rightarrow \infty} \sum_{n=0}^{N} n I_{n-1}^{*}\left(g_{n}(\cdot, \cdot)\right)=D_{\cdot}^{*} G \quad \text { in } L^{2}(v), \\
\lim _{N \rightarrow \infty} \lim _{\ell \rightarrow \infty} G^{N, \ell} & =G \quad \text { in } L^{2}(\Omega) .
\end{aligned}
$$

Since $\mathrm{d} \mu, \mathrm{d} \lambda \ll \mathrm{d} \nu$, it follows that

$$
\begin{gathered}
\lim _{N \rightarrow \infty}\left\{\lim _{k \rightarrow \infty} \lim _{\ell \rightarrow \infty} \mathrm{E}\left\{\delta\left(u^{N, k}\right) G^{N, \ell}\right\}\right\}=\mathrm{E}\left\{\delta^{*}(u) G\right\}, \\
\lim _{N \rightarrow \infty}\left\{\lim _{k \rightarrow \infty} \lim _{\ell \rightarrow \infty} \mathrm{E}\left\{\int_{0}^{1} u_{t}^{N, k}\left(D_{t} G^{N, \ell}\right) \mathrm{d}[M, M]_{t}\right\}\right\}=\mathrm{E}\left\{\int_{0}^{1} u_{t}\left(D_{t}^{*} G\right) \mathrm{d}[M, M]_{t}\right\}, \\
\lim _{N \rightarrow \infty}\left\{\lim _{k \rightarrow \infty} \lim _{\ell \rightarrow \infty} \mathrm{E}\left\{\int_{0}^{1} u_{t}^{N, k}\left(D_{t} G^{N, \ell}\right) \mathrm{d} t\right\}\right\}=\mathrm{E}\left\{\int_{0}^{1} u_{t}\left(D_{t}^{*} G\right) \mathrm{d} t\right\} .
\end{gathered}
$$

Therefore it suffices to prove (5.33) for $u=u^{N, k}$ and $G=G^{N, \ell}$. Note that in this case $\delta^{*}(u)=\delta(u)$, and $D^{*} G=D . G$, so by the definition of $\delta(u)(4.3)$ we have

$$
\mathrm{E}\left\{\delta^{*}(u) G\right\}=\mathrm{E}\left\{\delta\left(u^{N, k}\right) G^{N, \ell}\right\}=\mathrm{E}\left\{\int_{0}^{1} u_{t}^{N, k}\left(D_{t} G^{N, \ell}\right) \mathrm{d} t\right\}=\mathrm{E}\left\{\int_{0}^{1} u_{t} D_{t}^{*} G \mathrm{~d} t\right\}
$$

On the other hand, since all $f_{m}^{k}$ and $g_{n}^{\prime}$ are symmetric, we have by Lemma 5.2 that 


$$
\begin{aligned}
\mathrm{E}\left\{\int_{0}^{1} u_{t}\left(D_{t}^{*} G\right) \mathrm{d}[M, M]_{t}\right\} & =\mathrm{E}\left\{\sum_{m, n=0}^{N} \int_{0}^{1} I_{m}\left(f_{m}^{k}(\cdot, t)\right) n I_{n-1}\left(g_{n}^{\prime}(\cdot, t)\right) \mathrm{d}[M, M]_{t}\right\} \\
& =\sum_{m, n=0}^{N} n\left(f_{m}^{k}, g_{n}^{\prime}\right)_{m+1, n}=\sum_{n=1}^{N} n(n-1) !\left\langle f_{n-1}^{k}, g_{n}^{\prime}\right\rangle_{n} \\
& =\sum_{m, n=0}^{N} \mathrm{E}\left\{I_{m+1}\left(f_{m}^{k}\right) I_{n}\left(g_{n}^{\prime}\right)\right\}=\mathrm{E}\left\{\delta^{*}(u) G\right\} .
\end{aligned}
$$

Therefore (5.33) holds, proving the theorem.

\section{A class of stochastic differential equations}

In this section we study a class of SDEs based on the anticipating stochastic integrals discussed in the previous sections. Notice that since we have not yet derived any path regularity for the anticipating integral, the traditional ways of dealing with SDEs will not apply here. The method that we will use relies solely on the structure of the CRP of the $L^{2}$ processes and the definition of anticipating integrals, hence it will also be valid for the Brownian case. However, in the Brownian case much better results are available; see, for example, Buckdahn (1994).

We consider the following linear SDE for $0 \leqslant t \leqslant 1$ :

$$
X_{t}=H_{t}+\int_{0}^{t} \alpha(s) X_{s} \delta M_{s}+\int_{0}^{t} \beta(s) X_{s} \mathrm{~d} s,
$$

where $H_{t}$ is a square-integrable process, and $\alpha$ and $\beta$ are deterministic functions. Since we have no knowledge of the path regularity of the anticipating integral, equation (6.1) is understood to hold in $L^{2}(\mathrm{~d} t \times \mathrm{d} P)$.

We shall make use of the following assumptions on the process $H$ :

Assumption 6.1. The process $H$ is decomposable as $H=G+J$, where $J$ is an adapted, square-integrable process, and $G$ belongs to a subspace of $L^{2}([0,1] \times \Omega)$ consisting of a finite number of chaoses. In other words, $G=\sum_{n=1}^{N} I_{n}\left(g_{n}(\cdot, t)\right)$.

Assumption 6.2. The functions $\alpha$ and $\beta$ are bounded, measurable and non-random.

We shall denote $\|\alpha\|:=\|\alpha\|_{\infty}$, and similarly for $\beta$.

Observe that by linearity of the equation, every solution of (6.1) can be written as the sum $X=X^{0}+X^{1}$, where $X^{0}$ and $X^{1}$ are the solutions of the following SDEs, respectively:

$$
X_{t}^{0}=J_{t}+\int_{0}^{t} \alpha(s) X_{s}^{0} \mathrm{~d} M_{s}+\sum_{0}^{t} \beta(s) X_{s}^{0} \mathrm{~d} s ;
$$


and

$$
X_{t}^{1}=G_{t}+\int_{0}^{t} \alpha(s) X_{s}^{1} \delta M_{s}+\int_{0}^{t} \beta(s) X_{s}^{1} \mathrm{~d} s .
$$

Note that by the semimartingale theory (cf., for example, Protter 1990), (6.2) always possesses a unique adapted solution that is square-integrable, so we need pay attention only to equation (6.3). To simplify the representation, let us first assume that $\beta \equiv 0$. That is to say, we first consider a simpler form of (6.3):

$$
X_{t}=G_{t}+\int_{0}^{t} \alpha(s) X_{s} \delta M_{s}
$$

Assume that $X$ is a solution of (6.4), and write $X_{t}=\sum_{n=0}^{\infty} I_{n}\left(f_{n}(\cdot, t)\right), \quad G_{t}=$ $\sum_{n=0}^{N} I_{n}\left(g_{n}(\cdot, t)\right)$, where $f_{n}, g_{n} \in L^{2}\left(T^{n+1}\right)$ and for almost all $t \in[0,1], f_{n}(\cdot, t), g_{n}(\cdot, t) \in$ $L_{s}^{2}\left(T^{n}\right), n=1,2, \ldots$ Then it is necessary that

$$
\sum_{n=0}^{\infty} I_{n}\left(f_{n}(\cdot, t)\right)=\sum_{n=0}^{N} I_{n}\left(g_{n}(\cdot, t)\right)+\sum_{n=0}^{\infty} I_{n+1}\left(\overline{\left.f_{n}(\cdot, *)\right) \alpha(*) 1_{[0, t]}(*)^{\mathrm{s}}}\right),
$$

by the definition of the anticipating stochastic integral. Comparing both sides of (6.5) term by term, we see that

$$
\begin{aligned}
f_{n}(\cdot, *, t) & = \begin{cases}g_{n}(\cdot, *, t)+\overline{f_{n-1}(\cdot, *) \alpha(*) 1_{[0, t]}(*)^{\mathrm{s}}}, & 1 \leqslant n \leqslant N, \\
\overline{f_{n-1}(\cdot, *) \alpha(*) 1_{[0, t]}(*)^{\mathrm{s}}}, & n>N,\end{cases} \\
f_{0}(t) & =g_{0}(t),
\end{aligned}
$$

where, the symbol ' ' represents $n-2$ variables, while '*' represents one variable. Therefore, we have reduced the problem to finding a sequence $\left\{f_{n}\right\}$ such that (6.6) is satisfied and every term in (6.4) defined by using $\left\{f_{n}\right\}$ makes sense. Note that (6.6) is a recursive procedure, so the sequence $\left\{f_{n}\right\}$ is uniquely determined. Since any solution to (6.4) must satisfy (6.6), we conclude that the equation (6.4) possesses at most one solution.

It now remains to show the existence of the solution to (6.4). By the preceding argument, we assume that the sequence $\left\{f_{n}\right\}$ is defined via (6.6). Since $N>0$ is a fixed, finite integer, the values of the terms $f_{0}, f_{1}, \ldots, f_{N}$ will not affect the convergence of the sums appearing in (6.5). We shall only analyse the values of $\left\{f_{n}\right\}_{n \geqslant N+1}$. Let us first define some notation. For each $p \geqslant 1$, let $\mathbb{P}^{N+p}$ denote all permutations of $\{1, \ldots, N+p\}$, and

$$
\mathscr{A}_{p}:=\left\{\sigma \in \mathbb{P}^{N+p} \mid \sigma(i)=i, i \notin\{\sigma(N+k), N+k: k=1, \ldots, p\}\right\} ;
$$

that is, $\mathscr{A}_{p}$ consists of those permutations $\sigma$ that keep indices $i$ unchanged unless $i \geqslant N+1$ or it is the image of $N+k$ under $\sigma$, for some $p \geqslant k \geqslant 1$. Define, for each $p \geqslant 1$,

$$
\mathscr{B}_{p}:=\left\{\sigma \in \mathscr{C}_{p} \mid \sigma(N+1)<\sigma(N+2)<\ldots<\sigma(N+p)\right\} .
$$

Denote, for any finite set $\mathscr{S}$, by $|\mathscr{S}|$ the cardinality of $\mathscr{S}$. 
Lemma 6.1. If $\mathscr{A}_{p}, \mathscr{S}_{p}, p=1,2, \ldots$ are defined by (6.7) and (6.8), then

$$
\left|\mathscr{C}_{p}\right|=\prod_{k=1}^{p}(N+k), \quad\left|\mathscr{B}_{p}\right|=\left(\begin{array}{c}
N+p \\
p
\end{array}\right) .
$$

Consequently, $\left|\mathscr{C}_{p}\right| /\left|\mathscr{B}_{p}\right|=p !$.

Proof. We proceed by induction. For $p=1$, note that the typical element in $\mathscr{A}_{1}$ is of the form:

$$
\left(\begin{array}{cccccccc}
1 & 2 & \ldots & j-1 & j & j+1 & \ldots & N+1 \\
1 & 2 & \ldots & j-1 & N+1 & j+1 & \ldots & j
\end{array}\right), \quad j=1, \ldots, N+1 .
$$

Therefore it is easily seen that $\left|\mathscr{b}_{1}\right|=N+1$. Also, in this case, we have $\left|\mathscr{B}_{1}\right|=\left|\mathscr{C}_{1}\right|=N+1$, so (6.9) is proved.

Next, assume that $\mathscr{b}_{1}, \ldots, \mathscr{C}_{k}$ are constructed so that (6.9) holds, and consider the case when $p=k+1$. Define, for each $j=1, \ldots, N+k+1$, and $\sigma \in \mathscr{A}_{k}$, a permutation $\sigma^{j}$ by

$$
\sigma^{j}(i)= \begin{cases}j, & i=N+k+1, \\ N+k+1, & \sigma(i)=j \\ \sigma(i), & \text { otherwise. }\end{cases}
$$

Then we have $\mathscr{C}_{k+1}=\bigcup_{j=1}^{N+k+1} \mathscr{C}_{k}^{j}$, where $\mathscr{C}_{k}^{j}:=\left\{\sigma^{j} \mid \sigma \in \mathscr{b}_{k}\right\}$. Note that for every $j$, we have $\left|\mathscr{C}_{k}^{j}\right|=\left|\mathscr{C}_{k}\right|$ and $\mathscr{C}_{k}^{j} \cap \mathscr{C}_{k}^{\ell}=\varnothing$ for $j \neq \ell$; thus

$$
\left|\mathscr{b}_{k+1}\right|=\sum_{j=1}^{N+k+1}\left|\mathscr{C}_{k}^{j}\right|=(N+k+1)\left|\mathscr{C}_{k}\right|=(N+k+1) \prod_{j=1}^{N+k}(N+j)=\prod_{j=1}^{N+k+1}(N+j),
$$

proving the lemma.

Our next goal is to take a closer look at the sequence $\left\{f_{n}\right\}$ obtained by solving (6.6), so that an estimation can be made to prove the $L^{2}$-convergence of the series appearing in (6.5).

Lemma 6.2. Let $\left\{f_{n}\right\}$ be the sequence defined by (6.6). Then, for any $p \geqslant 1$,

$$
\begin{aligned}
f_{N+p}\left(t_{1}, \ldots, t_{N+p}, t\right)= & \frac{1}{\left|\mathscr{C}_{p}\right|} \sum_{\sigma \in \mathscr{C}_{p}} f_{N}\left(t_{\sigma(1)}, \ldots, t_{\sigma(N+1)}\right) \\
& \times\left\{\prod_{i=1}^{p-1} \alpha\left(t_{\sigma(N+i)}\right) 1_{\left[0, t_{\sigma(N+i+1)}\right.}\left(t_{\sigma(N+i)}\right)\right\} \alpha\left(t_{\sigma(N+p)}\right) 1_{[0, t]}\left(t_{\sigma(N+p)}\right) .
\end{aligned}
$$


Proof. We again proceed by induction. For $p=1$, using Lemma 6.1 we have

$$
\begin{aligned}
f_{N+1}\left(t_{1}, \ldots, t_{N+1}, t\right) & =\overline{f_{N}\left(t_{1}, \ldots, t_{N+1}\right) \alpha\left(t_{N+1}\right) 1_{[0, t]}\left(t_{N+1}\right)^{\mathrm{s}}} \\
& =\frac{1}{N+1} \sum_{i=1}^{N+1} f_{N}\left(t_{1}, \ldots, t_{i-1}, t_{N+1}, t_{i+1}, \ldots, t_{N}, t_{i}\right) \alpha\left(t_{i}\right) 1_{[0, t]}\left(t_{i}\right) \\
& =\frac{1}{\left|\mathscr{C}_{1}\right|} \sum_{\sigma \in \mathscr{C}_{1}} f_{N}\left(t_{\sigma(1)}, \ldots, t_{\sigma(N+1)}\right) \alpha\left(t_{\sigma(N+1)}\right) 1_{[0, t]}\left(t_{\sigma(N+1)}\right) .
\end{aligned}
$$

Thus (6.11) is true. Now suppose that (6.11) is true for $N+p$. We have by definition of the symmetrization and (6.6) that

$$
\begin{aligned}
& f_{N+p+1}\left(t_{1} \ldots, t_{N+p+1}, t\right) \\
& =\frac{1}{N+p+1} \sum_{j=1}^{N+p+1} f_{N+p}\left(t_{1}, \ldots, t_{j-1}, t_{N+p+1}, t_{j+1}, \ldots, t_{N+p}, t_{j}\right) \alpha\left(t_{j}\right) 1_{[0, t]}\left(t_{j}\right) .
\end{aligned}
$$

Let us define

$$
\bar{t}_{i}^{j}:= \begin{cases}t_{i}, & i \neq j, N+p+1, \\ t_{N+p+1}, & i=j, \\ t_{j}, & i=N+p+1 .\end{cases}
$$

This, together with the induction hypothesis, enables us to write the summands on the righthand side of (6.12) as

$$
\begin{aligned}
f_{N+p}\left(\bar{t}_{1}^{j}, \ldots, \bar{t}_{N+p}^{j}\right)= & \frac{1}{\left|\mathscr{C}_{p}\right|} \sum_{\sigma \in \mathscr{b}_{p}} f_{N}\left(\bar{t}_{\sigma(1)}^{j}, \ldots, \bar{t}_{\sigma(N+1)}^{j}\right) \\
& \times \prod_{k=1}^{p} \alpha\left(\bar{t}_{\sigma(N+k)}^{j}\right) 1_{\left[0, \bar{t}_{\sigma(N+k+1)}^{i}\right]}\left(\bar{t}_{\sigma(N+p)}^{j}\right) .
\end{aligned}
$$

We show that, for any $\sigma \in \mathscr{A}_{p}$,

$$
\bar{t}_{\sigma(k)}^{j}=t_{\sigma^{j}(k)}, \quad k=1, \ldots, N+p,
$$

where $\sigma^{j}$ is defined by (6.10). In fact, by (6.10) and (6.13) we have, for any $j, t_{j}=$ $t_{\sigma^{j}(N+p+1)}$; and for those $k$ such that $\sigma(k)=j$ we have $\bar{t}_{\sigma(k)}^{j}=\bar{t}_{j}^{j}=t_{N+p+1}=t_{\sigma^{j}(k)}$. Finally, for those $k$ such that $\sigma(k) \neq j$ we have $\bar{t}_{\sigma(k)}^{j}=t_{\sigma(k)}$. Hence (6.15) is proved.

Now noting that $\left|\mathscr{C}_{p+1}\right|=(N+p+1)\left|\mathscr{A}_{p}\right|$, we have from (6.11), (6.12) (6.14) and (6.15) that

$$
\begin{aligned}
& f_{N+p+1}\left(t_{1}, \ldots, t_{N+p+1}, t\right) \\
& \left.\left.=\frac{1}{N+p+1} \sum_{j=1}^{N+p+1} \frac{1}{\left|\mathscr{C}_{p}\right|} \sum_{\sigma \in \mathscr{b}_{p}} f_{N}\left(\bar{t}_{\sigma(1)}^{j}, \ldots, \bar{t}_{\sigma(N+1)}^{j}\right) \prod_{k=1}^{p} \alpha\left(\bar{t}_{\sigma(N+k)}^{j}\right) 1_{\left[0, \bar{t}_{\sigma(N+k+1)}^{i}\right]}\right] \bar{t}_{\sigma(N+p)}^{j}\right) \\
& \quad \times \alpha\left(t_{j}\right) 1_{[0, t]}\left(t_{j}\right)
\end{aligned}
$$




$$
\begin{aligned}
= & \frac{1}{\left|\ell_{p+1}\right|} \sum_{j=1}^{N+p+1} \sum_{\sigma^{j} \in \ell^{j}} f_{N}\left(t_{\sigma^{j}(1)}, \ldots, t_{\sigma^{j}(N+1)}\right) \prod_{k=1}^{p} \alpha\left(t_{\sigma^{j}(N+k)}\right) 1_{\left[0, t_{\sigma} \mathrm{j}_{(N+k+1)}\right]}\left(t_{\sigma^{j}(N+p)}\right) \\
& \times \alpha\left(t_{\sigma^{j}(N+p+1)}\right) 1_{[0, t]}\left(t_{\sigma^{j}(N+p+1)}\right) \\
= & \frac{1}{\left|\mathscr{b}_{p+1}\right|} \sum_{\sigma \in \ell_{p+1}} f_{N}\left(t_{\sigma(1)}, \ldots, t_{\sigma(N+1)}\right) \prod_{k=1}^{p} \alpha\left(t_{\sigma(N+k)}\right) 1_{\left[0, t_{\sigma(N+k+1)}\right]}\left(t_{\sigma(N+p)}\right) \\
& \times \alpha\left(t_{\sigma(N+p+1)}\right) 1_{[0, t]}\left(t_{\sigma(N+p+1)}\right),
\end{aligned}
$$

since $\mathscr{C}_{p+1}=\bigcup_{j=1}^{N+p+1} \mathscr{A}_{p}^{j}$. Therefore (6.11) holds for $N+p+1$ and the proof is now complete.

In order to prove the $L^{2}$-convergence of the series $X_{t}=\sum_{n} I_{n}\left(f_{n}(\cdot, t)\right)$, we first evaluate the $L^{2}$-norms of the $f_{n}$ on the set $\Sigma_{n}$. Again, we consider only the terms $f_{n}, n>N$. Suppose that $\left(t_{1}, \ldots, t_{N+p}\right) \in \Sigma_{N+p}$. Since $t_{1}<t_{2}<\ldots<t_{N+p}$, we have by definition of the set $\mathscr{B}_{p}$ and some simple computations that

$$
f_{N+p}\left(t_{1}, \ldots, t_{N+p}\right)=\frac{1}{\left|\mathscr{C}_{p}\right|} \sum_{\sigma \in \mathscr{S}_{p}} f_{N}\left(t_{\sigma(1)}, \ldots, t_{\sigma(N+1)}\right)\left\{\prod_{i=1}^{p} \alpha\left(t_{\sigma(N+i)}\right)\right\} 1_{[0, t]}\left(t_{\sigma(N+p)}\right) .
$$

Define, for each $p \geqslant 1$ and $\sigma \in \mathscr{B}_{p}$,

$$
f_{N, p}^{\sigma}=f_{N}\left(t_{\sigma(1)}, \ldots, t_{\sigma(N+1)}\right)\left\{\prod_{i=1}^{p} \alpha\left(t_{\sigma(N+i)}\right)\right\} 1_{[0, t]}\left(t_{\sigma(N+p)}\right)
$$

Then under Assumption 6.2 we have

$$
\begin{aligned}
\left\|f_{N, p}^{\sigma}\right\|_{L^{2}\left(\Sigma_{N+p}\right)}^{2} & =\int_{\Sigma_{N+p}}\left|f_{N, p}^{\sigma}\right|^{2} \mathrm{~d} t_{1} \ldots \mathrm{d} t_{N+p} \\
& \leqslant\|\alpha\|^{2 p}\left\|f_{N}\right\|_{N}^{2} \int_{t_{\sigma(N+1)}<\ldots<t_{\sigma(N+p)}<t} \mathrm{~d} t_{\sigma(N+1)} \ldots \mathrm{d} t_{\sigma(N+p)} \\
& =\frac{\|\alpha\|^{2 p}\left\|f_{N}\right\|_{N}^{2} \cdot t^{p}}{p !}
\end{aligned}
$$

Therefore, by (6.15) we have 


$$
\begin{aligned}
\left\|f_{N+p}(\cdot, t)\right\|_{N+p}^{2} & =(N+p) !\left\|f_{N+p}(\cdot, t)\right\|_{L^{2}\left(\Sigma_{N+p}\right)}^{2} \\
& \leqslant(N+p) !\left\{\frac{1}{\left|\mathscr{C}_{p}\right|} \sum_{\sigma \in \mathscr{S}_{p}}\left\|f_{N, p}^{\sigma}\right\|_{L^{2}\left(\Sigma_{N+p}\right)}\right\}^{2} \\
& \leqslant(N+p) !\left(\frac{\left|\mathscr{B}_{p}\right|}{\left|\mathscr{C}_{p}\right|}\right)^{2}\left\|f_{N, p}^{\sigma}\right\|_{L^{2}\left(\Sigma_{N+p)}\right)}^{2} \\
& \leqslant \frac{(N+p) !}{(p !)^{3}}\|\alpha\|^{2 p}\left\|f_{N}\right\|_{N}^{2} \cdot t^{p} .
\end{aligned}
$$

Now let us define, for each $n \in \mathbb{N}, M_{n}:=\sum_{k=0}^{n}\left\|I_{k}\left(f_{k}(\cdot, \cdot)\right)\right\|_{L^{2}(T \times \Omega)}^{2}$, and $C:=\left\|f_{N}\right\|_{L^{2}\left(T^{N}\right)}^{2}$. Noting that

$$
\frac{(N+p) !}{p !}=\left(1+\frac{N}{p}\right)\left(1+\frac{N}{p-1}\right) \ldots(1+N) N ! \leqslant(1+N)^{p} N !,
$$

we deduce that

$$
\begin{aligned}
\mathrm{E} \int_{0}^{1}\left|X_{t}\right|^{2} \mathrm{~d} t & =\int_{0}^{1} \sum_{p=1}^{\infty}\left\|I_{N+p}\left(f_{N+p}(\cdot, t)\right)\right\|_{L^{2}(\Omega)}^{2} \mathrm{~d} t+\int_{0}^{1} \sum_{k=0}^{N}\left\|I_{k}\left(f_{k}(\cdot, t)\right)\right\|_{L^{2}(\Omega)}^{2} \mathrm{~d} t \\
& =\int_{0}^{1} \sum_{p=1}^{\infty}(N+p) !\left\|f_{N+p}(\cdot, t)\right\|_{N+p}^{2} \mathrm{~d} t+M_{N} \\
& \leqslant C \sum_{p=1}^{\infty} \frac{((N+p) !)^{2}}{(p !)^{3}}\|\alpha\|^{2 p} \int_{0}^{1} t^{p} \mathrm{~d} t+M_{N} \\
& \leqslant C(N !)^{2} \sum_{p=1}^{\infty} \frac{(1+N)^{2 p}}{(p+1) !}\|\alpha\|^{2 p}+M_{N} \\
& \leqslant C(N !)^{2} \mathrm{e}^{(1+N)^{2}\|\alpha\|^{2}}+M_{N}<\infty
\end{aligned}
$$

Therefore, $X \in L^{2}(\mathrm{~d} t \times \mathrm{d} P)$. It remains to check that for each $t \in[0,1]$ the process $X . \alpha(\cdot) 1_{[0, t]}(\cdot) \in \operatorname{Dom}(\delta)$. By Lemma 4.6, it suffices to show that

$$
\sum_{n}\left\|I_{n+1}\left(f_{n}(\cdot, *) \alpha(*)\right)\right\|_{L^{2}(\Omega)}^{2}<\infty
$$

Since for each $p \geqslant 1$ we have by (6.17) that 


$$
\begin{aligned}
\left\|I_{N+p+1}\left(f_{N+p}(\cdot, *) \alpha(*)\right)\right\|_{L^{2}(\Omega)}^{2} & \leqslant(N+p+1) ! \int_{0}^{1}\left\|f_{N+p}(\cdot, s)\right\|_{N+p}^{2} \alpha^{2}(s) \mathrm{d} s \\
& \leqslant C(N+p+1) !\|\alpha\|^{2(p+1)}\left\{\frac{(N+p) !}{(p !)^{3}} \int_{0}^{1} s^{p} \mathrm{~d} s\right\} \\
& \leqslant C \frac{(N+p+1) !(N+p) !}{(p !)^{2}(p+1) !}\|\alpha\|^{2(p+1)} .
\end{aligned}
$$

Now an estimation similar to before shows that

$$
\begin{aligned}
\sum_{n=0}^{\infty}\left\|I_{n+1}\left(f_{n}(\cdot, *) \alpha(*)\right)\right\|_{L^{2}(\Omega)}^{2} & =\sum_{p=1}^{\infty}\left\|I_{N+p+1}\left(f_{N+p+1}(\cdot, *) \alpha(*)\right)\right\|_{L^{2}(\Omega)}^{2}+M_{N+1} \\
& \leqslant C(N+1)\|\alpha\|^{2}(N !)^{2} \mathrm{e}^{\|\alpha\|^{2}(N+1)^{2}}+M_{N+1}<\infty .
\end{aligned}
$$

Thus (6.18) holds, and we have proved the following theorem:

Theorem 6.3. Suppose that Assumptions 6.1 and 6.2 hold. Then the SDE (6.4) has a unique solution $X_{t}=\sum_{n=0}^{\infty} I_{n}\left(f_{n}(\cdot, t)\right)$, where $\left\{f_{n}\right\}_{n=0}^{\infty}$ satisfies the recursive equations (6.6).

We now consider the general case when $\beta \neq 0$. Since the argument is virtually the same, we give only an outline. Suppose Assumptions 6.1 and 6.2 hold. It is easy to check that in this case the recursive equation (6.6) should be replaced by

$$
\begin{aligned}
& f_{n}(\cdot, *, t)= \begin{cases}g_{n}(\cdot, *, t)+\overline{f_{n-1}(\cdot, *) \alpha(*) 1_{[0, t]}(*)^{\mathrm{s}}}+\int_{0}^{t} f_{n}(\cdot, *, s) \beta(s) \mathrm{d} s & n \leqslant N, \\
\overline{f_{n-1}(\cdot, *) \alpha(*) 1_{[0, t]}(*)^{\mathrm{s}}}+\int_{0}^{t} f_{n}(\cdot, *, s) \beta(s) \mathrm{d} s & n>N,\end{cases} \\
& f_{0}(t)=g_{0}(t)+\int_{0}^{t} f_{0}(s) \beta(s) \mathrm{d} s .
\end{aligned}
$$

The integral equation $x(t)=\eta(t)+\int_{0}^{t} x(s) \beta(s) \mathrm{d} s$ has the unique solution

$$
x(t)=\eta(t)-\int_{0}^{t} \mathrm{e}^{-\int_{s}^{t} \beta(u) \mathrm{d} u} \beta(s) \eta(s) \mathrm{d} s .
$$

Therefore, if we denote $\gamma(s, t)=\mathrm{e}^{-\int_{s}^{t} \beta(u) \mathrm{d} u} \beta(s)$, and

$$
h_{n}(\cdot, *, t)= \begin{cases}\left.g_{n}(\cdot, *, t)+\overline{f_{n-1}(\cdot, *) \alpha(*) 1_{[0, t]}(*)}\right)^{\mathrm{s}} & n \leqslant N, \\ \overline{f_{n-1}(\cdot, *) \alpha(*) 1_{[0, t]}(*)^{\mathrm{s}}} & n>N,\end{cases}
$$


then from (6.19) we have

$$
\begin{gathered}
f_{0}(t)=g_{0}(t)-\int_{0}^{t} \gamma(s, t) g_{0}(s) \mathrm{d} s, \\
f_{n}(\cdot, *, t)=h_{n}(\cdot, *, t)-\int_{0}^{t} \gamma(s, t) h_{n}(\cdot, *, s) \mathrm{d} s .
\end{gathered}
$$

Again we need only consider the terms when $n>N$. Denoting $m(s, t):=$ $\alpha(s)\left(1-\int_{0}^{s} \gamma(u, t) \mathrm{d} u\right)$, we show by induction that the sequence $\left\{f_{n}\right\}$ defined by (6.19) satisfies, for any $p \geqslant 1$,

$$
\begin{gathered}
f_{N+p}\left(t_{1}, \ldots, t_{N+p}, t\right)=\frac{1}{\left|\mathscr{b}_{p}\right|} \sum_{\sigma \in \ell_{p}} f_{N}\left(t_{\sigma(1)}, \ldots, t_{\sigma(N+1)}\right) \\
\times\left\{\prod_{i=1}^{p-1} m\left(t_{\sigma(N+i)}, t_{\sigma(N+i+1)}\right) 1_{\left[0, t_{\sigma(N+i+1)}\right.}\left(t_{\sigma(N+i)}\right)\right\} m\left(t_{\sigma(N+p)}, t\right) 1_{[0, t]}\left(t_{\sigma(N+p)}\right) .
\end{gathered}
$$

Indeed, for $p=1$, using Lemma 6.1 and the similar computation in Lemma 6.2, we have

$$
\begin{aligned}
f_{N+1}\left(t_{1}, \ldots, t_{N+1}, t\right) & =\frac{1}{N+1} \sum_{i=1}^{N+1} f_{N}\left(\begin{array}{c}
t_{i} \\
t_{N+1}
\end{array}\right) \alpha\left(t_{i}\right) 1_{[0, t]}\left(t_{i}\right)\left\{1-\int_{0}^{t} \gamma(s, t) 1_{[0, s]}\left(t_{i}\right) \mathrm{d} s\right\} \\
& =\frac{1}{\left|\mathscr{C}_{1}\right|} \sum_{\sigma \in \mathscr{C}_{1}} f_{N}\left(t_{\sigma(1)}, \ldots, t_{\sigma(N+1)}\right) m\left(t_{\sigma(N+1)}, t\right) 1_{[0, t]}\left(t_{\sigma(N+1)}\right)
\end{aligned}
$$

where

$$
f_{N}\left(\begin{array}{c}
t_{i} \\
t_{N+1}
\end{array}\right):=f_{N}\left(t_{1}, \ldots, t_{i-1}, t_{N+1}, t_{i+1}, \ldots, t_{N}, t_{i}\right) .
$$

Therefore (6.20) holds for $p=1$. Now suppose that (6.20) is true for $N+p$. We have

$$
\begin{aligned}
f_{N+p+1}\left(t_{1}, \ldots, t_{N+p+1}, t\right) \\
=\frac{1}{N+p+1} \sum_{j=1}^{N+p+1} f_{N+p}\left(t_{1}, \ldots, t_{j-1}, t_{N+p+1}, t_{j+1}, \ldots, t_{N+p}, t_{j}\right) m\left(t_{j}, t\right) 1_{[0, t]}\left(t_{j}\right) \\
=\frac{1}{N+p+1} \sum_{j=1}^{N+p+1}\left\{\frac{1}{\left|\mathscr{b}_{p}\right|} \sum_{\sigma \in \ell_{p}} f_{N}\left(\bar{t}_{\sigma(1)}^{j}, \ldots, \bar{t}_{\sigma(N+1)}^{j}\right)\right. \\
\left.\quad \times \prod_{k=1}^{p} m\left(\bar{t}_{\sigma(N+k)}^{j}, \bar{t}_{\sigma(N+k+1)}^{j}\right) 1_{\left[0, \bar{t}_{\sigma(N+k+1)}^{j}\right]}\left(\bar{t}_{\sigma(N+k)}^{j}\right) \cdot m\left(\bar{t}_{\sigma(N+p+1)}^{j}, t\right) 1_{[0, t]}\left(\bar{t}_{N+p+1}^{j}\right)\right\}
\end{aligned}
$$




$$
\begin{aligned}
= & \frac{1}{\left|\mathscr{b}_{p+1}\right|} \sum_{\sigma \in \ell_{p+1}} f_{N}\left(t_{\sigma(1)}, \ldots, t_{\sigma(N+1)}\right) \\
& \times \prod_{k=1}^{p+1} m\left(t_{\sigma(N+k)}, t_{\sigma(N+k+1)}\right) 1_{\left[0, t_{\sigma(N+p+2)}\right]}\left(t_{(\sigma(N+p+1)}\right),
\end{aligned}
$$

where $t_{\sigma(N+p+2)}=t$, proving $(6.20)$.

Note that the boundedness of $\alpha$ and $\beta$ implies that $m$ is also bounded, so by replacing $\|\alpha\|_{\infty}$ by $\|m\|_{\infty}$ in the estimations in the case $\beta=0$, we obtain the main result of this section:

Theorem 6.4. Suppose that Assumptions 6.1 and 6.2 hold. Then the SDE (6.3) has a unique solution $X_{t}=\sum_{n=0}^{\infty} I_{n}\left(f_{n}(\cdot, t)\right)$, where $\left\{f_{n}\right\}_{n=0}^{\infty}$ satisfies the recursive equations (6.19).

We can combine Theorem 6.4 with the semimartingale theory to obtain:

Theorem 6.5. Suppose that Assumptions 6.1 and 6.2 hold. Then equation (6.1) has a solution and it is unique in $L^{2}(\mathrm{~d} t \times \mathrm{d} P)$.

Proof. We only show the uniqueness. Let $X^{0}$ be the unique semimartingale solution of (6.2); and let $Y$ be another solution of (6.1). Let

$$
\mathscr{C}(Z):=\int_{0}^{t} \alpha(s) Z_{s} \delta M_{s}+\int_{0}^{t} \beta(s) Z_{s} \mathrm{~d} s, \quad \forall Z \in L^{2}(\mathrm{~d} t \times \mathrm{d} P) .
$$

Thus we have $Y=H+\mathscr{B}(Y)$ and

$$
Y-X^{0}=H+\mathscr{C}(Y)-\left(J+\mathscr{C}\left(X^{0}\right)\right)=H-J+\mathscr{L}\left(Y-X^{0}\right)=G+\mathscr{B}\left(Y-X^{0}\right),
$$

and by Theorem 6.4 we have $Y-X^{0}=X^{1}$, the unique solution of (6.3). So $Y=X^{0}+X^{1}$ in $L^{2}(\mathrm{~d} t \times \mathrm{d} P)$ and we have uniqueness.

\section{Acknowledgement}

The first author was supported in part by NSF grant DMS 9301516 and ONR grant N0001496-1-0262. The second author was supported in part by NSF grant 9401109-INT, NSA grant 904-94-H-2049 and ONR grant N00014-96-1-0262. The third author wishes to thank the Mathematics Department of Purdue University for its hospitality, during which much of the research for this paper was carried out. We also thank two anonymous referees for suggestions that improved the paper. 


\section{References}

Barlow, M. (1981) Construction of a martingale with given absolute value. Ann. Probab., 9, 314-320. Buckdahn, R. (1994) Anticipative Girsanov Transformations and Skorohod Stochastic Differential equations, Mem. Amer. Math. Soc., 111 (533).

Dellacherie, C. (1972) Capacités et Processus Stochastiques. Berlin: Springer-Verlag.

Dellacherie, C., Maisonneuve, B. and Meyer, P.A. (1992) Probabilités et Potentiel: Chapitres XVII à $X X I V$. Paris: Hermann.

Emery, M. (1989) On the Azéma martingales. In J. Azéma, P.A. Meyer and M. Yor (eds), Sém. de Probab. XXIII, Lecture Notes in Math. 1372, pp. 66-87. New York: Springer-Verlag.

Kurtz, T.G. and Protter, P. (1991) Weak limit theorems for stochastic integrals and stochastic differential equations. Ann. Probab., 19, 1035-1070.

Meyer, P.A. (1976) Un cours sur les intégrales stochastiques. In P.A. Meyer (ed.), Sém. de Probab. X, Lecture Notes in Math. 511, pp. 245-400. New York: Springer-Verlag.

Meyer, P.A. (1989) Construction de solutions d'equations de structure. In J. Azéma, P.A. Meyer and M. Yor (eds), Sém. de Probab. XXIII, Lecture Notes in Math. 1372, pp. 142-145. New York: Springer-Verlag.

Meyer, P.A. (1993) Quantum Probability for Probabilistics, Lecture Notes in Math. 1538. New York: Springer-Verlag.

Nualart, D. and Pardoux, E. (1988) Stochastic calculus with anticipating integrands. Probab. Theory Related Fields, 78, 535-582.

Nualart, D. (1995) Malliavin Calculus and Related Topics. New York: Springer-Verlag.

Ocone, D. (1984) Malliavin calculus and stochastic integral representation of diffusion processes, Stochastics, 12, 161-185.

Protter, P. and Sharpe, M. (1979) Martingales with given absolute value. Ann. Probab., 7, 1056-1058.

Protter, P. (1990) Stochastic Integration and Differential Equations. Heidelberg: Springer-Verlag.

Russo, F. and Vallois, P. (1994) Product of two multiple stochastic integrals. Preprint.

Vallois, P. (1995) Decomposing the Brownian path via the range process. Stochastic Process. Appl., 55, 211-226.

Received February 1996 and revised January 1997. 